\title{
Identification of Hypoxia-Regulated Proteins Using MALDI-Mass Spectrometry Imaging Combined with Quantitative Proteomics
}

Djidja, Marie-Claude; Chang, Joan; Hadjiprocopis, Andreas; Schmich, Fabian; Sinclair, John; Mrsnik, Martina; Schoof, Erwin M.; Barker, Holly E.; Linding, Rune; Jørgensen, Claus

Total number of authors:

11

Published in:

Journal of Proteome Research

Link to article, DOI:

$10.1021 /$ pr401056c

Publication date:

2014

Document Version

Publisher's PDF, also known as Version of record

Link back to DTU Orbit

Citation (APA):

Djidja, M-C., Chang, J., Hadjiprocopis, A., Schmich, F., Sinclair, J., Mrsnik, M., Schoof, E. M., Barker, H. E., Linding, R., Jørgensen, C., \& Erler, J. T. (2014). Identification of Hypoxia-Regulated Proteins Using MALDi-Mass Spectrometry Imaging Combined with Quantitative Proteomics. Journal of Proteome Research, 13, 2297-2313. https://doi.org/10.1021/pr401056c

\section{General rights}

Copyright and moral rights for the publications made accessible in the public portal are retained by the authors and/or other copyright owners and it is a condition of accessing publications that users recognise and abide by the legal requirements associated with these rights.

- Users may download and print one copy of any publication from the public portal for the purpose of private study or research.

- You may not further distribute the material or use it for any profit-making activity or commercial gain

- You may freely distribute the URL identifying the publication in the public portal 


\section{a \\ pubs.acs.org/jpr

\section{Identification of Hypoxia-Regulated Proteins Using MALDI-Mass Spectrometry Imaging Combined with Quantitative Proteomics}

Marie-Claude Djidja, ${ }^{\dagger, \#,}$ Joan Chang, ${ }^{\dagger, \|, \bullet, \perp}$ Andreas Hadjiprocopis, ${ }^{\dagger}$ Fabian Schmich, ${ }^{\dagger,}{ }_{\text {John Sinclair, }}{ }^{\dagger}$ Martina Mršnik, ${ }^{\dagger,}$ Erwin M. Schoof, ${ }^{\S}$ Holly E. Barker, ${ }^{\dagger}$ Rune Linding, ${ }^{\S}$ Claus Jørgensen, ${ }^{\ddagger}$ and Janine T. Erler* $*,+\|, \perp$

${ }^{\dagger}$ Hypoxia and Metastasis Team and ${ }^{\ddagger}$ Cell Communications Team, Cancer Research U.K. Tumour Cell Signalling Unit,
Division of Cancer Biology, The Institute of Cancer Research, London, United Kingdom
${ }^{\S}$ Cellular Signal Integration Group (C-SIG), Centre for Biological Sequence Analysis, Department of Systems Biology,
Technical University of Denmark, DK-2800 Lyngby, Denmark
${ }^{\perp}$ Biotech Research and Innovation Centre (BRIC), University of Copenhagen, Ole Maaløes Vej 5, Copenhagen 2200, Denmark

Supporting Information

ABSTRACT: Hypoxia is present in most solid tumors and is clinically correlated with increased metastasis and poor patient survival. While studies have demonstrated the role of hypoxia and hypoxia-regulated proteins in cancer progression, no attempts have been made to identify hypoxiaregulated proteins using quantitative proteomics combined with MALDImass spectrometry imaging (MALDI-MSI). Here we present a comprehensive hypoxic proteome study and are the first to investigate changes in situ using tumor samples. In vitro quantitative mass spectrometry analysis of the hypoxic proteome was performed on breast cancer cells using stable isotope labeling with amino acids in cell culture (SILAC). MS analyses were performed on laser-capture microdissected samples isolated from normoxic and hypoxic regions from tumors derived from the same cells used in vitro. MALDI-MSI was used in combination to investigate hypoxia-regulated

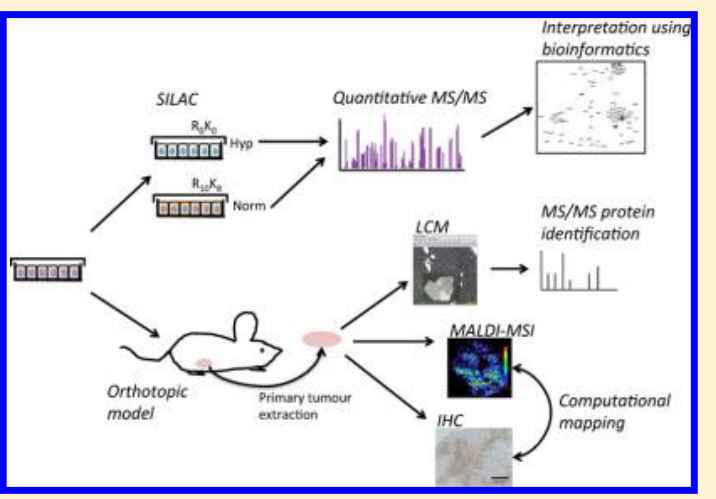
protein localization within tumor sections. Here we identified more than 100 proteins, both novel and previously reported, that were associated with hypoxia. Several proteins were localized in hypoxic regions, as identified by MALDI-MSI. Visualization and data extrapolation methods for the in vitro SILAC data were also developed, and computational mapping of MALDI-MSI data to IHC results was applied for data validation. The results and limitations of the methodologies described are discussed.

KEYWORDS: hypoxia, mass spectrometry imaging, proteomics

\section{INTRODUCTION}

Hypoxia arises when solid tumors outgrow their blood supplies and additionally develop abnormal vasculature systems. ${ }^{1}$ This results in localized regions in the tumor with poor delivery of oxygen and lack of nutrients. Hypoxia has been shown to be clinically correlated with metastasis, treatment failure, and decreased cancer patient survival. ${ }^{2}$ Studies have reported the increased invasiveness ability and metastatic potential of hypoxic tumor cells, ${ }^{3}$ and it has long been known that hypoxia tumor cells are resistant to radiotherapy and many forms of chemotherapy. ${ }^{4}$ Understanding protein changes that mediate metastatic progression and drug resistance is of great interest for understanding disease progression and improving therapeutic efficacy. ${ }^{4 \mathrm{~b}}$ Such aims require powerful and robust tools as well as technologies that can provide insight into the molecular organization of the tumor microenvironment, such as to localize and identify proteins as well as to study proteinprotein interactions.

MALDI-MSI is rapidly becoming a powerful technology for studying the distribution as well as the in situ identification of several classes of compounds directly within biological tissue sections with no requirement of predefined targets. ${ }^{5}$ It is therefore possible to visualize the distribution of multiple key molecules and investigate their relationship with the tumor microenvironment or with drugs administered. ${ }^{6}$ It hence creates new translational opportunities in cancer research and provides large and heterogeneous data sets for the study of potential cellular and molecular networks present within tumor tissue sections, enabling in-depth studies of the tumor microenvironment. ${ }^{7}$ In brief, tissue sections are uniformly coated with a matrix prior to data acquisition. In most cases, the image acquisition is performed by irradiating the sample under a stationary laser at a spatial resolution predefined by the operator and the instrument capacities. The recorded mass spectra are then processed together using imaging software, and ion density maps are generated by plotting the $x$ and $y$ coordinates against the relative abundance or intensity of a given

Received: October 23, 2013

Published: April 7, 2014 
analyte within the tissue section. Several studies have reported MALDI-MSI as a huge potential for translational research technology for cancer biomarker discovery. ${ }^{4 \mathrm{~b}, 8}$ Development strategies including sample preparation methodologies plus instrumental development have been reported and demonstrated improvement in MALDI-MSI techniques.

The use of MALDI-MSI has been found to be powerful for the direct analysis of in situ digested proteins within both frozen and formalin-fixed paraffin embedded (FFPE) tissue sections, leading to the in situ identification of several proteins within the tissue sections. ${ }^{9}$ Although MALDI-MSI is a powerful technology for the study of the localization of several biomolecule classes directly within tissue sections, its quantitative nature is still limited.

While several studies have reported the strong association of hypoxia and metastasis, none have performed quantitative proteomic studies of hypoxia-regulated proteins and combined this type of data with MALDI-MSI. Here we present a comprehensive quantitative proteomic study combined with MALDI-MSI to identify hypoxia-regulated proteins both in vitro and in situ. Quantitative methodologies were developed via the stable isotope labeling with amino acid in cell culture (SILAC). SILAC-labeled breast cancer cells were grown under normoxic and hypoxic conditions and analyzed with mass spectrometric identification and relative quantification of the identified peptides. LC-MS/MS analyses were also conducted on regions of normoxia and hypoxia of breast cancer tumors grown from the same cells used in vitro isolated by laser capture microdissection (LCM). Together, this allowed the identification of over a thousand proteins, including more than 100 unique hypoxia-regulated proteins. MALDI-MSI allowed the localization and further identification/validation of hypoxiaregulated proteins at primary tumor sites. The results and limitations of the methodologies used here are discussed.

\section{MATERIAL AND METHODS}

\section{Stable Isotope Labeling of Proteins of 4T1breast Cancer Cells}

Dialyzed FBS and penicillin/streptomycin were obtained from GibcoBRL/Invitrogen. Dulbecco's modified Eagle's medium (DMEM) was obtained from Caisson lab, and 'heavy' and 'light' arginine (R10, R0) and lysine (K8, K0) were obtained from Sigma. Metastatic mouse mammary cancer 4T1 cells (a kind gift from Fred Miller ${ }^{10}$ ) underwent SILAC according to a protocol previously described by Jørgensen et al. ${ }^{11}$ In brief, SILAC media were prepared with $10 \%$ dialyzed FBS, penicillin/ streptomycin and $50 \mathrm{mg} / \mathrm{L}$ of arginine and lysine. Cells were cultured at $37{ }^{\circ} \mathrm{C}$ under normoxic conditions $\left(5 \% \mathrm{CO}_{2}\right.$ and $20 \% \mathrm{O}_{2}$ ) for at least 10 passages to ensure complete protein labeling. Labeling efficacy was determined prior to experimental procedures to ensure cells were at least $95 \%$ labeled. Cells were plated onto $8.5 \mathrm{~cm}^{2}$ tissue culture dishes (Invitrogen) and grown to $\sim 60 \%$ confluency before hypoxic or normoxic treatment for $24 \mathrm{~h}$.

\subsection{In Vitro Hypoxic Conditions}

For hypoxic treatment, the 4T1 mammary cancer cells were placed into a Hypoxystation (Don Whitley Scientifics) and incubated at $37{ }^{\circ} \mathrm{C}$ with $5 \% \mathrm{CO}_{2}$ and $1 \% \mathrm{O}_{2}$. The hypoxic treatment was carried out for $24 \mathrm{~h}$.
Protein Extraction and In-Gel Digestion

Following incubation, hypoxic and normoxic cells were washed with ice-cold TBS and lysed with urea buffer (8 M urea, $100 \mathrm{mM}$ Tris- $\mathrm{HCl} \mathrm{pH}$ 8, Sigma-Aldrich, Dorset, U.K.). Protein concentrations were determined via bicinchoninic assay (BCA) (BCA Protein Assay kit, Thermo Fisher Scientific). A small aliquot of each sample was kept to check for full incorporation of labeled amino acids, and the remaining lysates were mixed in a one-to-one ratio. Thirty $\mu \mathrm{g}$ of the mixture was run on $10 \%$ SDS-PAGE (NuPAGE, Invitrogen) at $190 \mathrm{~V}$ for $1 \mathrm{~h}$ The gel was then stained with InstantBlue (Expedeon), and 10 gel bands were excised.

The gel pieces were washed sequentially with $50 \mathrm{mM}$ triethylamine bicarbonate (TEAB) buffer (Sigma-Aldrich) and dehydrated in ACN (Sigma-Aldrich) until they were completely destained. In-gel protein reduction and alkylation were carried out using $2 \mathrm{mM}$ tris(2-carboxyethyl)phosphine hydrochloride (Sigma-Aldrich) at $55{ }^{\circ} \mathrm{C}$ for $30 \mathrm{~min}$ and $55 \mathrm{mM}$ iodoacetamide (Sigma-Aldrich) at room temperature for $30 \mathrm{~min}$, respectively. Tryptic digestion was performed at $37^{\circ} \mathrm{C}$ for $16 \mathrm{~h}$ using $20 \mu \mathrm{g} / \mathrm{mL}$ of trypsin (Promega, Southampton, U.K.) contained in $50 \mathrm{mM}$ TEAB buffer. The extract digest solutions were transferred to clean vials, dried in a speedvac (Thermo SAVANT SC250 Exp SpeedVac Concentrator), and stored at $-80{ }^{\circ} \mathrm{C}$ until LC-MS/MS analysis.

\section{Tumor Samples}

All animal procedures and handling was approved by the U.K. Home Office (PPL 6796) and performed following UKCCCR Guidelines for the Welfare and use of animals in cancer research. ${ }^{12}$ 4T1 mammary cancer cells were resuspended in PBS prior to injection. One $\times 10^{5}$ cells were injected into the fourth mammary fat pad of Balb/c mice (Harlan Laboratories). Three mice were used for this study. Mice were monitored and culled when tumors reached the maximum allowed size (about 4 weeks after tumor cell injection). For this study, five tumor samples were collected and used. One hour prior to sacrifice, mice were injected intraperitoneally with $100 \mu \mathrm{L}$ of pimonidazole hydrochloride (pimo) (hydroxyprobe-1; NPI, Belmont, MA) at $60 \mathrm{mg} / \mathrm{kg}$. Tumour tissue samples were harvested and immediately treated with a Stabilizor system (Denator $\mathrm{AB}$, Sweden) according to the supplier instructions. This treatment aimed to inhibit enzymatic activity within the tissue sample. The tumor tissue samples were fixed in $4 \%$ buffered formalin and embedded in paraffin. Alternatively, tumors were snap-frozen in liquid nitrogen and stored at $-80{ }^{\circ} \mathrm{C}$ until further analysis. Five $\mu \mathrm{m}$ sections were cut using either a cryostat (Leica Microsystems, U.K.) or cryotome (Leica Microsystems, U.K.) and mounted onto indium tin oxide (ITO) (Sigma-Aldrich) or histological glass slides (Menzel-Glaser, Germany). Formalin-fixed paraffin-embedded (FFPE) tissue sections were stored at room temperature until further analysis.

\section{Laser Capture Microdissection, Protein Extraction and Digestion}

For LCM, $5 \mu \mathrm{m}$ frozen or FFPE tumor tissue sections were cut using a cryostat (Leica Microsystems, U.K.) and mounted on DIRECTOR glass slides (Expression Pathology). The tissue sections were dehydrated and stained with hematoxylin according to a protocol provided by Expression Pathology. Immunohistochemical staining for hypoxia markers pimo and CA-IX were also performed on serial tissue sections for visual localization of hypoxic and normoxic regions. The LCM was 
carried out using a LEICA LMD6000 (LEICA Microsystems). Approximately $10^{6}$ cells from hypoxic and normoxic regions were collected into eppendorf caps. In solution protein reduction, alkylation and tryptic digestion were performed according to the Expression Pathology protocol. The peptides were desalted and purified using C-18 stage tip (3 M Empore) and then dried in a speedvac (Thermo SAVANT SC250 Exp SpeedVac Concentrator) and stored at $-80{ }^{\circ} \mathrm{C}$ until LC-MS/MS analysis.

\section{Tissue Preparation for MALDI-MSI}

Prior to trypsin and matrix deposition, FFPE tumor tissue sections were prepared as previously described. ${ }^{12}$ In brief, paraffin film was removed using xylene; then, the tissue sections were rehydrated in a series of ethanol solutions. Antigen retrieval was performed in AR buffer (DAKO) in a water bath for $15 \mathrm{~min}$ at $90{ }^{\circ} \mathrm{C}$. The sections were cooled to room temperature, rinsed with water, and then allowed to dry at room temperature before trypsin and matrix deposition.

Trypsin and matrix depositions were achieved using the ImagePrep station (Bruker Daltonik, Bremen, Germany). A trypsin solution was prepared at $20 \mu \mathrm{g} / \mathrm{mL}$ in $50 \mathrm{mM}$ ammonium bicarbonate buffer $(\mathrm{pH} 8.1)$ containing $0.1 \%$ octyl glucoside. ${ }^{13}$ Using the ImagePrep station, $400 \mu \mathrm{L}$ of trypsin solution was sprayed onto the tissue section over 60 spraying cycles, thus ensuring that the tissue section is not over wetted. The tissue section was then incubated for $2 \mathrm{~h}$ at $37{ }^{\circ} \mathrm{C}\left(5 \% \mathrm{CO}_{2}\right)$ in a humid environment. Alpha-cyano-4hydrocinnamic acid (Sigma-Aldrich), mixed with aniline (Sigma-Aldrich), was used as matrix. ${ }^{13,14}$ The matrix solution was prepared at $7 \mathrm{mg} / \mathrm{mL}$ in $60 \% \mathrm{ACN}$ and $0.1 \%$ TFA and deposited onto the tissue sections using the ImagePrep station.

NanoLC-MS/MS Analyses and Database Searching

Trypsin-digested peptides from both LCM and in-gel digestion were subjected to LC-MS/MS using the NanoLC-Ultra 2D chromatography system (Eksigent, Dublin, Ireland) coupled to an LTQ Orbitrap Velos mass spectrometer (Thermo Fisher Scientific, Hemel Hempstead, U.K.). Reversed-phase chromatographic separation was carried out on a $50 \mu \mathrm{m}$ inner diameter $\times$ $100 \mathrm{~mm}$ C18 ReproSil-Pur column (3 $\mu \mathrm{m}$ particle size; Dr Maisch HPLC, Germany) over a 40 min linear gradient of 5$40 \%$ solvent B (solvent A: $0.1 \%$ formic acid; solvent B: ACN $100 \%, 0.1 \%$ formic acid). The mass spectrometer was operated in the data-dependent mode to automatically switch between Orbitrap MS and ion trap MS/MS acquisition. Survey full-scan MS spectra (from $\mathrm{m} / z$ 375-2000) were acquired in the Orbitrap with a resolution of 60000 at $\mathrm{m} / z 400$ and FT target value of $1 \times 10^{6}$ ions. The 20 most abundant ions were selected for CID and dynamically excluded for $8 \mathrm{~s}$. For accurate mass measurement, the lock mass option was enabled using the polydimethylcyclosiloxane ion $(m / z 455.120025)$ as an internal calibrant.

For protein identification from LCM samples, raw MS/MS data were submitted for database searching using Proteome Discoverer v1.3 and Mascot v2.2. and searched against SwissProt version 57.14 (514789 sequences) mammalian (64919 entries) and Mus musculus (1622 entries). The following Mascot search parameters were used: peptide tolerance, $10 \mathrm{ppm}$; fragment tolerance, $0.6 \mathrm{Da}$; trypsin missed cleavage, 2; variable modifications, acetyl (Protein N-term) carbamidomethyl (C), oxidation of methionine, PyroGlu (Peptide N-term Q). MS/MS-based peptide and protein identifications were grouped and validated using Scaffold v3.0
(Proteome Software, Portland, OR). Protein identifications were automatically accepted if they contained at least two unique peptides assigned with at least $95 \%$ confidence by Peptide Prophet1, in accordance with guidelines from Journal of Proteome Research.

For peptide identification from in-gel digested samples, raw MS/MS data files were submitted for database searching using Proteome Discoverer v1.3 and Mascot v2.2 and searched against Swiss-Prot (Sprot_Mouse_20110414.fasta, v14-3-3) as well as Ensembl (EN_mmusR53_fasta, version EC 1.6.5.3). Another database was used to facilitate the STRING Network analysis. The following MASCOT search parameters were used: peptide tolerance, $100 \mathrm{ppm}$; fragment tolerance, $0.6 \mathrm{Da}$; trypsin missed cleavage, 2; variable modifications, acetyl (protein $\mathrm{N}$-term) carbamidomethyl (C), oxidation of methionine, PyroGlu (Peptide N-term Q). For MS/MS-derived in vitro SILAC data, the following modifications were added: SILAC Lys $\left({ }^{13} \mathrm{C}_{6}{ }^{15} \mathrm{~N}_{2}\right)$ SILAC Arg $\left({ }^{13} \mathrm{C}_{6}{ }^{15} \mathrm{~N}_{4}\right)$.

\section{MALDI-MSI and MALDI-MS/MS Analyses}

MALDI-MSI analyses were acquired directly from the in situ digested tumor sections over a mass range $\mathrm{m} / z 800$ to 2500 in reflector positive mode using a 4800 Plus MALDI TOF/TOF analyzer (Applied Biosystems/MDS Sciex, Concord, Canada) operating with a $200 \mathrm{~Hz} \mathrm{Nd:YAG} \mathrm{laser.} \mathrm{The} \mathrm{instrument} \mathrm{calibra-}$ tion was performed using a solution of peptide standards (4700 Proteomics analyzer calibration mixture, Applied Biosystems/ MDS Sciex) ranging between $\mathrm{m} / z 900$ and 4000 prior to MALDI-MSI analysis. Peptide images were acquired at a spatial resolution set at $80 \mu \mathrm{m}$ with 250 laser shots per position, and ion images were generated with TissueView 1.0 software (Applied Biosystems/MDS Sciex), which can be used to measure the amplitude of selected mass signals and to reconstruct $2 \mathrm{D}$ ion density maps.

MALDI-MS/MS analyses were performed directly from the in situ digested tumor tissue sections. Using the control software, the tissue section areas were subdivided into small partitions to mimic an LC-MALDI plate across the tissue sections. MS data were acquired at $200 \mathrm{~Hz}$ with 200 laser shots/spec. Subspectra were acquired every 50 shots. Automatic MS/MS data were acquired at $200 \mathrm{~Hz}$ in $1 \mathrm{kV}$ MS/MS mode with 2000 laser shots/spectrum on the top three precursors. For peptide identification from on-tissue digested tissues, MS/MS peak lists were submitted for database searching using Mascot v2.2 and searched against Swiss-Prot (Sprot_Mouse_20110414.fasta, v14-3-3) as well as Ensembl (EN_mmusR53_fasta, version EC 1.6.5.3). The following MASCOT search parameters were used: peptide tolerance, $100 \mathrm{ppm}$; fragment tolerance, 0.6 Da; trypsin missed cleavage, 2; variable modifications, acetyl (protein $\mathrm{N}$-term) carbamidomethyl (C), oxidation of methionine, PyroGlu (peptide N-term Q).

\section{Network Analysis Using PANTHER and STRING}

Data sets obtained from SILAC in vitro quantitative studies, which contained hypoxia-regulated proteins, were classified according to biological process using the PANTHER platform (available from www.pantherdb.org).

In addition to PANTHER analysis, the SILAC data sets were analyzed using STRING database version 9.0. ${ }^{15}$ The STRING database was queried about the associations of all MS-detected proteins with a combined confidence score of at least 0.8 . The pipeline is implemented by several computer programs implemented in Perl (Perl version 5.14.2) and Bash (GNU Bash version 4.0.33) scripts. It is fully automated, ensuring that 
the results are completely reproducible and free of bias. In brief, the pipeline was constructed as follows: conversion of the MS results spreadsheet into text files; creation of a mapping between Ensembl and HUGO protein identification by enquiring Biomart (http://www.biomart.org); and, if required, STRING using its web API (http://string-db.org/help/index. jsp?topic=/org.string-db.docs/api.html). The purpose of this is to use Ensembl identification for STRING queries and HUGO gene symbols for generating protein interaction networks with readily understandable protein names; MS-detected proteins were then grouped according to significance (significance defined as $>1.4$ fold change between hypoxia and normoxia); STRING was queried about interactions between the proteins, and the interactions without a high enough STRING score were filtered out; a graph was constructed where the nodes are the significant proteins and the edges are the interactions as returned by STRING; two lists were generated for each node in the graph, one of its immediate neighbors with significant upregulation and one of its immediate neighbors with significant down-regulation, allowing data extrapolation for ease of analysis; and statistics of the resulting graph such as degree distribution, clustering coefficient, and so on were calculated. These can be used to programmatically identify hubs, networks, and proteins bridging the networks and correlate these results with fold changes in protein expression.

The final step in the pipeline is to convert the graph into Cytoscape $2.8^{16}$ data and respective property files for interactive visualization using the following conventions:

1) Each node represents a protein detected by MS. Note: kinases are depicted as squares to distinguish from other types of proteins.

2) The color of each node represents various properties of the protein detected:

a. Red: up-regulated in hypoxia. Intensity of color correlates with increase in up-regulation.

b. Blue: down-regulated in hypoxia. Intensity of color correlates with increase in down-regulation.

c. White: no significant changes between the two conditions.

3) The size of each node represents its degree; the degree is defined as the number of associations the protein has.

4) Proteins with no interactions satisfying the specified minimum STRING score are removed from the resulting figure.

Validation of Hypoxia-Associated Proteins Using Statistical Analysis of MALDI-MSI Data

The detailed methodology used here can be found in the Supporting Information (Method S1). In brief, MALDI-MSI scans were subjected to spectral processing including baseline correction, peak identification, and peak alignment prior to statistical analysis. For statistical analyses, MALDI-MS images were coregistered with IHC stained images. An example of coregistered image is shown in Figure S1 in the Supporting Information. Aligned MS peaks were extracted from hypoxia and normoxia regions, localized by IHC staining images, and tested for equal mean intensity using a $t$ test.

Immunofluorescence (IF) Staining of Pimonidazole on Frozen Pimo-Treated Tumor Tissue Sections

4T1 frozen pimo-treated tumor tissue sections were dried at room temperature for $30 \mathrm{~min}$ and then fixed in ice-cold acetone for $30 \mathrm{~min}$. Tissue sections were allowed to dry at room temperature for $20 \mathrm{~min}$, and an ImmEdge pen (H-4000, Vector Laboratories, Peterborough, U.K.) was used to encircle the sections, prior to incubation in $1 \% \mathrm{BSA} / \mathrm{PBS}$ for $30 \mathrm{~min}$ at room temperature. Sections were incubated with hydroxyprobe antipimo MAB-1 antibody (NPI, Belmont, MA) at a dilution of 1:100 in IF buffer for $1 \mathrm{~h}$ at room temperature in the dark. Control slides were incubated with just IF buffer during this period of time. This was followed by three washes with $300 \mu \mathrm{L}$ of PBS for $5 \mathrm{~min}$ each and then incubation with Goat antimouse AlexaFluor488 (Invitrogen) at 1:1000 dilution in IF buffer for $1 \mathrm{~h}$ at room temperature in the dark, followed by three washes with $300 \mu \mathrm{L}$ of PBS for 5 min each. The sections were then counter-stained with $200 \mu \mathrm{L}$ of $4^{\prime}, 6$-diamidino-2phenylindole (DAPI; D21490, Invitrogen,) at a dilution of 1:1000 in PBS. Slides were rinsed in PBS and mounted in Vectashield (H-1000, Vector Laboratories). The stained images were visualized using an Olympus BX51 research microscope (Olympus U.K., Southend-on-Sea, U.K.) with a DP72 camera (Olympus U.K.) operating in fluorescence mode and using CellD (Olympus U.K.) software.

\section{Immunohistochemistry (IHC) Staining on FFPE Tumor Tissue Sections}

Paraffin was removed from FFPE tumor tissue sections $(5 \mu \mathrm{m}$ thickness) by immersing the slides twice in $100 \%$ xylene (Sigma-Aldrich) at room temperature for $10 \mathrm{~min}$ each, followed by a rehydration of the tissue sections through a series of ethanol solutions of increasing water concentrations (100, 85, and $70 \%$; vol/vol in water, $10 \mathrm{~min}$ each) before a final $5 \mathrm{~min}$ of incubation in water. Residual endogenous peroxidase activity was blocked by incubating the sections in 3\% hydrogen peroxide in methanol for $15 \mathrm{~min}$ at room temperature. Antigen retrieval was carried out in a target retrieval citrate buffer $(\mathrm{pH}$ 6.0) (DAKO UK, Cambridgeshire, U.K.) at $95{ }^{\circ} \mathrm{C}$ for $15 \mathrm{~min}$. Sections were allowed to cool to room temperature, rinsed with $\mathrm{PBS}$, and then incubated in $\mathrm{PBS} / 5 \%$ goat blocking serum (Invitrogen) for $1 \mathrm{~h}$ at room temperature to minimize nonspecific binding. Sections were incubated with the following primary antibodies: hydroxyprobe antipimo MAB-1 antibody (NPI, Belmont, MA) at 1:100 dilution in PBS/1\% goat serum at room temperature for overnight. As negative staining controls, separate tissue section slides were incubated in PBS/1\% goat serum without primary antibodies. After washing with PBS, tumor tissue sections were then incubated with streptavidin-biotin peroxidase solution at 1:500 dilution (Vector Laboratories, Burlingame, CA) at room temperature for $30 \mathrm{~min}$. Visualization of antibody binding on the sections was performed using 3,3'-diaminobenzidine (BioGenex Laboratories, San Ramon, CA), and tissue sections were counterstained with Mayer's hematoxylin (Sigma Aldrich) for $60 \mathrm{~s}$ before being dehydrated and coverslipped. The images of the IHC staining were taken on an Olympus BX51 research microscope (Olympus U.K.) with a DP72 camera (Olympus U.K.) operating in brightfield mode using CellD (Olympus U.K.) software.

\section{RESULTS}

Quantitative Proteomic Analysis of Hypoxia-Associated Proteins in Vitro

Because hypoxia is prominent in solid tumors such as breast cancer and it was previously demonstrated that the HIF $1 \alpha$ levels have a positive correlation with pathologic stage, ${ }^{17}$ we 
A.

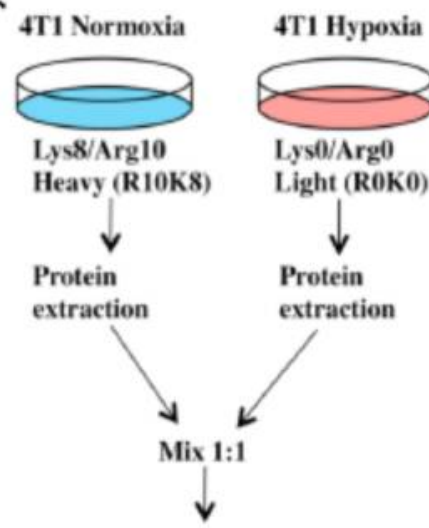

SDS PAGE

and in-gel

digestion

$\downarrow$

L.C.MS/MS

analysis on LTQ.

Velos Orbitrap

$\downarrow$

MASCOT

Database search
C.

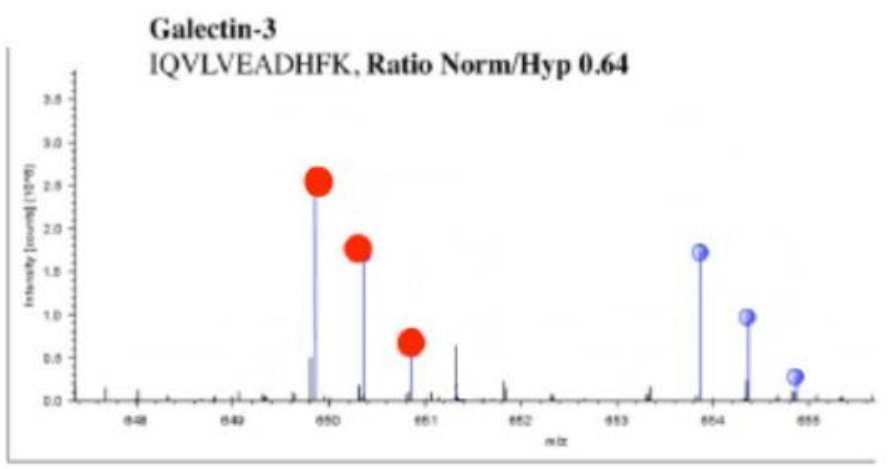

Phospho Glycerate Kinase

LGDVYVNDAFGTAHR, Ratio Norm/Hyp $\mathbf{0 . 6 4 5}$

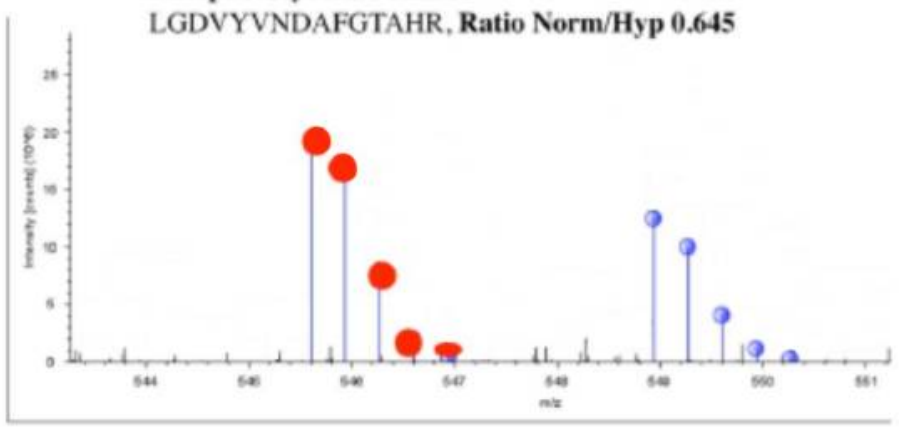

B.

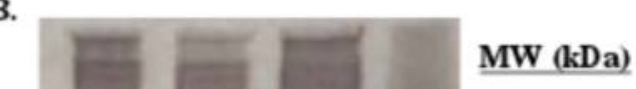

225

150

102

76

52

38

31

24

Figure 1. Experimental workflow for LC-MS/MS analysis of SILAC-labeled 4T1 breast cancer cells. (A) 4T1 mouse mammary cancer cells were incubated for $24 \mathrm{~h}$ in normoxia and hypoxia. Hypoxic cells were labeled with light arginine and lysine (R0K0) and normoxic cells were labeled with heavy arginine and lysine (R10K8). Cell populations were mixed in 1:1 ratio, and extracted proteins were separated on SDS-PAGE. Following in-gel digestion, peptides were isolated and purified prior to LC-MS/MS analysis on LTQ-Velos Orbitrap. (B) Digital scan of SDS-PAGE separation of protein extracts. (C) SILAC peptide pairs were relatively quantified for both hypoxic and normoxic conditions.

decided to focus our investigation on hypoxia-associated proteins in a breast cancer model.

To perform a quantitative study of the proteomic changes that occur in normoxic versus hypoxic breast cancer cells, we first used 4T1 mouse mammary cancer cells in vitro. These cells were chosen as we have previously shown that hypoxia drives metastasis of 4T 1 cells. ${ }^{18}$ The cells were grown independently in either the presence of 'light' arginine and lysine (ROK0) or 'heavy' arginine and lysine (R10K8) SILAC media. The R0K0 cells were subjected to $24 \mathrm{~h}$ of hypoxic treatment $\left(1 \% \mathrm{O}_{2}\right)$, and the R10K8 cells were subjected to $24 \mathrm{~h}$ normoxic treatment $\left(20 \% \mathrm{O}_{2}\right)$. Figure $1 \mathrm{~A}$ shows the experimental workflow for 
Table 1. List of Identified Significant Hypoxia-Regulated Proteins after LC-MS/MS Analysis of 4T1 SILAC-Labelled Mammary Cancer Cells ${ }^{a}$

\begin{tabular}{|c|c|c|c|c|c|}
\hline accession & description & A2: heavy/light & B2: heavy/light & C2: heavy/light & average heavy/light \\
\hline E9QLP8 & keratin, type I cytoskeletal 10 & 0.223 & 0.062 & 0.057 & 0.114 \\
\hline P05064 & fructose-bisphosphate aldolase A & 0.603 & 0.463 & 0.332 & 0.466 \\
\hline P12382 & 6-phosphofructokinase, liver type & 0.601 & 0.411 & 0.405 & 0.472 \\
\hline $\mathrm{P} 17751$ & triosephosphate isomerase & 0.573 & 0.476 & 0.384 & 0.478 \\
\hline P09411 & phosphoglycerate kinase 1 & 0.656 & 0.443 & 0.359 & 0.486 \\
\hline Q9DBJ1 & phosphoglycerate mutase 1 & 0.596 & 0.537 & 0.433 & 0.522 \\
\hline P06151 & L-lactate dehydrogenase A chain & 0.705 & 0.557 & 0.394 & 0.552 \\
\hline Q6IRU2 & tropomyosin alpha-4 chain & 0.484 & 0.835 & 0.517 & 0.612 \\
\hline F6TS28 & galectin-3 & 0.655 & 0.528 & 0.659 & 0.614 \\
\hline P16858 & glyceraldehyde-3-phosphate dehydrogenase & 0.777 & 0.59 & 0.489 & 0.619 \\
\hline P35700 & peroxiredoxin-1 & 0.438 & 0.78 & 0.709 & 0.642 \\
\hline P17182 & alpha-enolase & 0.812 & 0.666 & 0.512 & 0.663 \\
\hline $\mathrm{P} 16045$ & galectin-1 & 0.53 & 0.753 & 0.732 & 0.672 \\
\hline P07356 & annexin $\mathrm{A} 2$ & 0.846 & 0.518 & 0.682 & 0.682 \\
\hline Q6ZQ38 & cullin-associated NEDD8-dissociated protein 1 & 0.796 & 0.743 & 0.537 & 0.692 \\
\hline P97429 & annexin A4 & 0.894 & 0.573 & 0.621 & 0.696 \\
\hline \multicolumn{6}{|c|}{ Top 16 down-regulated proteins under hypoxic conditions } \\
\hline accession & description & A2: heavy/light & B2: heavy/light & C2: heavy/light & average heavy/light \\
\hline Q5SWU9 & acetyl-CoA carboxylase 1 & 1.587 & 1.547 & 1.26 & 1.465 \\
\hline P46935 & E3 ubiquitin-protein ligase NEDD4 & 1.763 & 1.221 & 1.443 & 1.476 \\
\hline Q61656 & probable ATP-dependent RNA helicase DDX5 & 1.544 & 1.292 & 1.645 & 1.494 \\
\hline F8WIE5 & E3 ubiquitin-protein ligase HECTD1 & 1.637 & 1.554 & 1.295 & 1.495 \\
\hline Q9ERU9 & E3 SUMO-protein ligase RanBP2 & 1.836 & 1.475 & 1.201 & 1.504 \\
\hline Q7TMY8-3 & isoform 3 of E3 ubiquitin-protein ligase HUWE1 & 2.172 & 1.355 & 1.041 & 1.523 \\
\hline E9QKG6 & ankyrin repeat domain-containing protein 17 & 1.591 & 1.66 & 1.439 & 1.563 \\
\hline Q7TMB8-2 & isoform 2 of cytoplasmic FMR1-interacting protein 1 & 2.282 & 1.285 & 1.176 & 1.581 \\
\hline Q8VDJ3 & vigilin & 2.026 & 1.498 & 1.376 & 1.633 \\
\hline Q9DCV7 & keratin, type II cytoskeletal 7 & 2.755 & 1.119 & 1.079 & 1.651 \\
\hline H3BJU7 & rho guanine nucleotide exchange factor 2 & 2.625 & 1.027 & 1.354 & 1.669 \\
\hline Q80U93 & nuclear pore complex protein Nup214 & 2.379 & 1.782 & 1.21 & 1.79 \\
\hline Q80TP3 & E3 ubiquitin-protein ligase UBR5 & 2.418 & 2.012 & 1.118 & 1.849 \\
\hline P67778 & prohibitin & 0.941 & 0.893 & 3.935 & 1.923 \\
\hline P10854 & histone $\mathrm{H} 2 \mathrm{~B}$ type $1-\mathrm{M}$ & 1.709 & 1.211 & 3.76 & 2.227 \\
\hline Q5XG71 & small subunit processome component 20 homologue & 6.601 & 1.68 & 3.08 & 3.787 \\
\hline
\end{tabular}

${ }^{a} 4 \mathrm{~T} 1$ mammary cancer cells were treated under normoxia and hypoxia conditions. Hypoxic cells were labelled with light arginine and lysine (R0K0) and normoxic cells were labelled with heavy arginine and lysine (R10K8). The samples were mixed 1:1 ratio and ran on SDS-PAGE followed by ingel digestion and LC-MS/MS analysis. Proteome Discoverer v1.3 and Mascot v2.2 were used to analyze the raw data files. A heavy/light ratio was calculated for each identified protein by the software. Significance was defined as at least 1.4-fold change in protein expression between the two conditions.

LC-MS/MS analysis of SILAC-labeled 4T1 mammary cancer cells. Proteins extracted from these two sets of cells were mixed 1:1 ratio, followed by SDS-PAGE separation. Figure 1B shows the digital scan of SDS-PAGE separation of the protein extracts from both individual and mixed 1:1 cell populations. After ingel digestion of the extracted proteins, analyses were conducted on the resulting peptides using LC-MS/MS (Figure 1C). It was confirmed that the labeled amino acids were incorporated $97.9 \%$ into the $4 \mathrm{~T} 1$ cells (data not shown).

Because the SILAC labeling methodology results in mass differences between peptides with similar sequences, it is possible to determine the relative abundance of each peptide and perform a quantitative comparison based on their extracted ion current (Figure 1C). Here LC-MS/MS analyses were repeated on three independent sets of SILAC-labeled 4T1 cells. In total, 1416 proteins were identified, 268 proteins were detected in all repeats, and 131 proteins were consistently upor down-regulated in all repeats. The complete protein list can be found in the Table S1 in the Supporting Information. 60 proteins were found to be consistently up-regulated under hypoxic conditions, including glyceraldehyde-3-phosphate dehydrogenase, L-lactate dehydrogenase A chain, and phosphoglycerate kinase. 71 proteins were found to be consistently down-regulated under hypoxic conditions. These included ribosomal proteins and ubiquitin-associated proteins. Table 1 shows a list of the most significant proteins found to be up- or down-regulated in hypoxic versus normoxic conditions (significance defined as $>1.4$ fold change between hypoxia and normoxia). Figure 2A shows the top up- or down-regulated proteins (based on fold change) in hypoxic versus normoxic conditions. The top 40 up- or down-regulated proteins were classified according to biological process and molecular functions using PANTHER. Figure $2 \mathrm{~B}$ shows the functional classification of the proteins that were found consistently up- or down-regulated under hypoxic conditions. It can be noted that both up- and down-regulated hypoxia-associated proteins are 


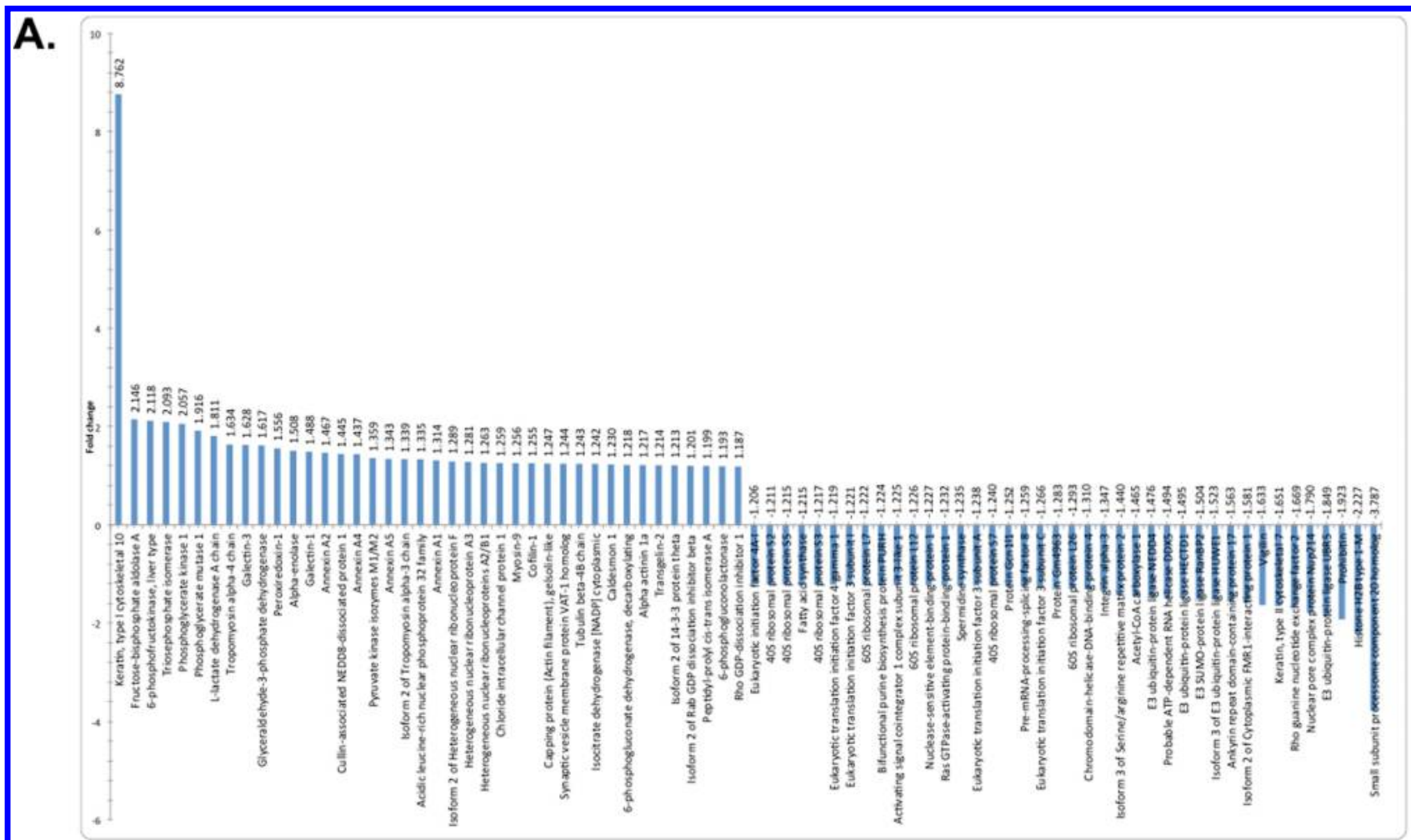

B.

b1)

4T1 (Up-regulated in hypoxia, biological processes)

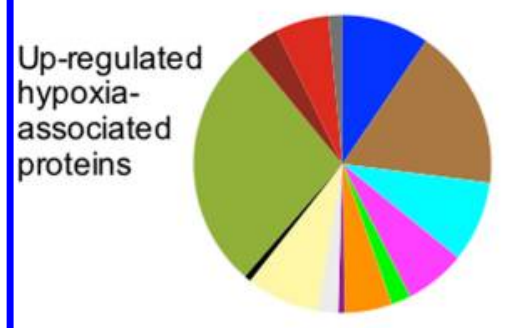

cell communication
cellular process
transport
cellular component organization
apoptosis
system process
reproduction
response to stimulus
developmental process
generation of precursor
metabolites and energy
metabolic process
immune system process

b3)

4T1 (Down-regulated in hypoxia, biological processes)

Down-

regulated

hypoxia-

associated

proteins

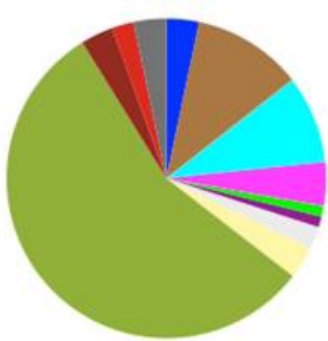

b2)

4T1 (Up-regulated in hypoxia, molecular functions)
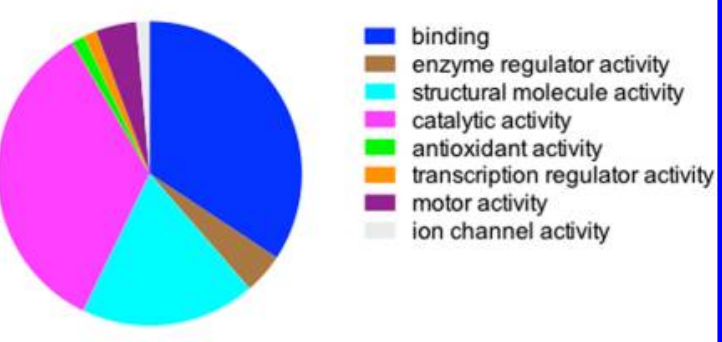

b4)

4T1 (Down-regulated in hypoxia, molecular functions)

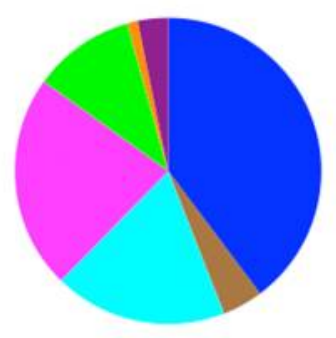

ainding

enzyme regulator activity

- structural molecule activity

- catalytic activity

- translation regulator activity

- transcription regulator activity

transporter activity

Figure 2. In vitro SILAC-LC-MS/MS quantification of proteins differentially regulated in hypoxia and normoxia in 4T1 cells. (A) Fold changes of regulated proteins in vitro in hypoxia versus normoxia. For quantitative analysis of the differences observed between peptide paired, a 1.4-fold cut off was applied. The top 40 up- and down-regulated hypoxia-associated proteins are displayed. The up-regulated proteins present in hypoxic regions are shown from the left and the down-regulated proteins in hypoxic regions are displayed from the right, with the fold-change displayed on top of the bars. (B) PANTHER classification of up and down-regulated hypoxia-associated proteins in vitro. Results from in vitro protein quantitation of 4T1 SILAC-labeled cells were analyzed using PANTHER software. Significantly up- and down-regulated hypoxia-associated proteins detected were classified according to (b1, b3) biological process, and (b2, b4) molecular function. 


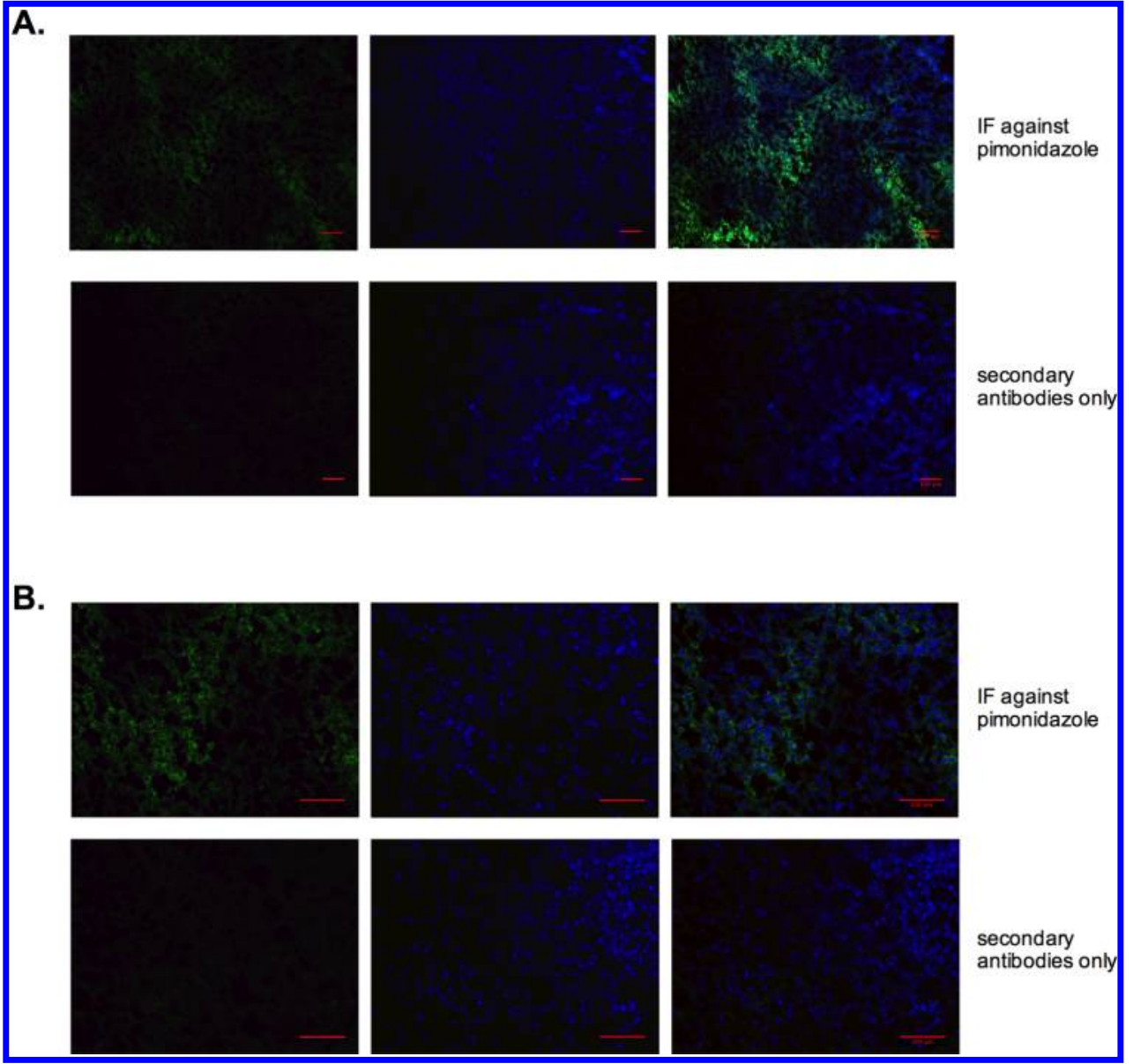

Figure 3. Immunofluorescence staining of Pimonidazole in frozen 4T1 primary mammary tumor tissue section. IF staining of pimo localization within frozen $4 \mathrm{~T} 1$ pimo-treated tumor tissue sections allows us to distinguish between hypoxic and normoxic regions for subsequent LCM-LC-MS/MS analyses. Left: pimo-immunofluorescence staining, middle: DAPI counter stain of cell nuclei, right: overlay of the two staining. Scale bars $=100 \mu \mathrm{m}$. Sections viewed at (A) $10 \times$ magnification and (B) $20 \times$ magnification.

mainly involved in biological processes like metabolic processes (e.g., up-regulation in protein and carbohydrate metabolic processes, down-regulation in protein and nucleobase/nucleoside/ nucleotide/nucleic acid metabolic processes) and cellular processes (e.g., up-regulation in cell communication, cell motion and reorganization of cellular components, downregulation in cellular component organization, cell cycle, cell adhesion, and cell communication), whereas the molecular functions of the proteins detected are mainly involved in binding (e.g., protein and nucleic acid binding in both cases, up-regulation in calcium ion and calcium-dependent phosphorlipid binding) and catalytic activities (e.g., up-regulation in oxidoreductase, transferase, hydrolase and isomerase activity, down-regulation in ligase, hydrolase and transferase activity).

Identification of Hypoxia-Associated Proteins in Situ Using LCM LC-MS/MS

Protein expression changes were also studied in situ using tissue samples isolated from frozen 4T1 tumor tissue sections. Regions of normoxia and hypoxia of orthotopic mammary tumors, grown from the same 4T1 cells as used in vitro, were isolated by laser microdissection (LCM). Pimonidazole (pimo), which is a small molecule used clinically to localize regions of hypoxia within tumor tissues, ${ }^{19}$ was used to locate hypoxic regions in our 4T1 tumor sections by IF (Figure 3). This allowed the visual localization of hypoxic and normoxic regions prior to LCM. The captured cells from each region of microdissected tumor tissue section were digested in solution and submitted to LC-MS/MS analysis. Following LC-MS/MS, 118 mouse proteins were identified within the normoxic LCM tissue sample, and 164 mouse proteins were identified within the hypoxic LCM tissue sample.

Tables S2 and S3 list the proteins identified in hypoxic regions and normoxic regions, respectively, and are given in the Supporting Information. The most readily detected proteins in the hypoxic regions were from a wide range of protein families including structural proteins (actins, myosins, collagens) and nuclear and translational proteins (histones, ribosomal proteins, elongation factors). When comparing both data sets obtained from LCM-LC-MS/MS analyses of hypoxic and normoxic regions, 89 proteins were found to be present in both hypoxic and normoxic regions, presenting either similar or different intensities. 75 proteins were detected only in hypoxic regions. Table 2 shows a summary list of the identified proteins from tissues isolated from in situ hypoxic regions of $4 \mathrm{~T} 1$ primary tumor sections after LCM-LC-MS/MS and which are associated with hypoxia. Standard and robust hypoxia-regulated proteins such as $78 \mathrm{kDa}$ glucose-regulated protein (Grp78), phosphoglycerate kinase, and L-lactate dehydrogenase A chain were found to be present in hypoxic regions. Grp78 was detected with a higher intensity in hypoxic regions compared with normoxic regions, whereas phosphoglycerate kinase and L-lactate dehydrogenase A chain were exclusively detected in 
Table 2. Summary List of Identified Proteins from Tissues Isolated from in Situ Hypoxic Regions of 4T1 Primary Tumour Sections after LCM-LC-MS/MS ${ }^{a}$

\begin{tabular}{|c|c|c|c|c|c|c|c|c|}
\hline \multicolumn{9}{|c|}{ 1. standard and robust hypoxia-regulated proteins } \\
\hline accession & description & \multirow{2}{*}{\multicolumn{2}{|c|}{$\begin{array}{l}\text { score } \\
16.75\end{array}$}} & coverage & no. proteins & peptide peak area & $\mathrm{MW}[\mathrm{kDa}]$ & \multirow{2}{*}{$\begin{array}{l}\text { peptide peak area found in normoxia } \\
\qquad 5.03 \times 10^{8}\end{array}$} \\
\hline P16045 & galectin-1 & & \multicolumn{2}{|c|}{23.7} & 1 & $2.09 \times 10^{8}$ & 14.9 & \\
\hline P20029 & $78 \mathrm{kDa}$ glucose-regulated protein & 49.2 & \multicolumn{2}{|c|}{19.08} & 1 & $5.93 \times 10^{7}$ & 72.4 & $3.48 \times 10^{7}$ \\
\hline P06151 & L-lactate dehydrogenase A chain & 11.8 & \multicolumn{2}{|c|}{10.24} & 2 & $2.55 \times 10^{7}$ & 36.5 & 0 (hypoxia only) \\
\hline P09411 & phosphoglycerate kinase 1 & 4.31 & \multicolumn{2}{|c|}{5.52} & 1 & $7.51 \times 10^{6}$ & 44.5 & 0 (hypoxia only) \\
\hline \multicolumn{9}{|c|}{ 2. Reported hypoxic regulation } \\
\hline accession & description & \multicolumn{2}{|r|}{ score } & coverage & \# proteins & peptide peak area & $\mathrm{MW}[\mathrm{kDa}]$ & peptide peak area found in normoxia \\
\hline P62204 & calmodulin & \multicolumn{2}{|r|}{40.12} & 30.87 & 2 & $1.36 \times 10^{8}$ & 16.8 & $1.08 \times 10^{8}$ \\
\hline P63101 & $14-3-3$ protein zeta/delta & \multicolumn{2}{|r|}{35.29} & 25.31 & 1 & $8.50 \times 10^{7}$ & 27.8 & $7.38 \times 10^{7}$ \\
\hline P48036 & annexin A5 & \multicolumn{2}{|r|}{29.19} & 19.75 & 1 & $6.94 \times 10^{7}$ & 35.7 & $4.18 \times 10^{7}$ \\
\hline P48678 & prelamin-A/C & \multicolumn{2}{|r|}{89.52} & 24.66 & 1 & $6.66 \times 10^{7}$ & 74.2 & 0 (hypoxia only) \\
\hline P18760 & cofilin-1 & & 14.08 & 31.93 & 1 & $6.38 \times 10^{7}$ & 18.5 & 0 (hypoxia only) \\
\hline P62259 & $14-3-3$ protein epsilon & & 33.04 & 25.88 & 1 & $5.78 \times 10^{7}$ & 29.2 & $5.44 \times 10^{7}$ \\
\hline P52480 & pyruvate kinase isozymes $\mathrm{M} 1 / \mathrm{M} 2$ & & 25.44 & 10.55 & 2 & $4.20 \times 10^{7}$ & 57.8 & $2.79 \times 10^{7}$ \\
\hline $\mathrm{P} 17182$ & alpha-enolase & & 20.48 & 10.14 & 1 & $3.96 \times 10^{7}$ & 47.1 & 0 (hypoxia only) \\
\hline P05064 & fructose-bisphosphate aldolase A & & 23.3 & 15.66 & 2 & $2.82 \times 10^{7}$ & 39.3 & 0 (hypoxia only) \\
\hline P08249 & malate dehydrogenase, mitochondrial & & 5.63 & 5.92 & 1 & $1.74 \times 10^{7}$ & 35.6 & $1.96 \times 10^{7}$ \\
\hline P14733 & lamin-B1 & & 14.77 & 9.35 & 1 & $1.74 \times 10^{7}$ & 66.7 & 0 (hypoxia only) \\
\hline P62962 & profilin-1 & & 6.1 & 17.14 & 1 & $1.35 \times 10^{7}$ & 14.9 & 0 (hypoxia only) \\
\hline P10107 & annexin $\mathrm{A} 1$ & & 13.27 & 11.85 & 2 & $1.29 \times 10^{7}$ & 38.7 & 0 (hypoxia only) \\
\hline O35639 & annexin $\mathrm{A} 3$ & & 10.65 & 11.76 & 1 & $1.29 \times 10^{7}$ & 36.4 & $5.70 \times 10^{6}$ \\
\hline H7BXC3 & triosephosphate isomerase & & 12.35 & 16.77 & 2 & $7.14 \times 10^{6}$ & 18 & $1.24 \times 10^{7}$ \\
\hline & 3. unreport & h) & ypoxic reg & gulation but & function ass & sociated with hypoxi & ia responses & \\
\hline accession & description & & score & coverage & $\begin{array}{c}\text { \# } \\
\text { proteins }\end{array}$ & peptide peak area & $\begin{array}{c}\mathrm{MW} \\
{[\mathrm{kDa}]}\end{array}$ & peptide peak area found in normoxia \\
\hline P10126 & elongation factor 1 -alpha 1 & & 41.39 & 18.4 & 1 & $1.73 \times 10^{8}$ & 50.1 & $1.17 \times 10^{8}$ \\
\hline P02089 & hemoglobin subunit beta- 2 & & 40.51 & 44.9 & 1 & $1.07 \times 10^{8}$ & 15.9 & $1.31 \times 10^{8}$ \\
\hline Q504P4 & heat shock cognate $71 \mathrm{kDa}$ protein & & 53.79 & 22.33 & 2 & $4.80 \times 10^{7}$ & 68.7 & $4.24 \times 10^{7}$ \\
\hline P11499 & heat shock protein HSP 90-beta & & 45.09 & 17.82 & 1 & $4.55 \times 10^{7}$ & 83.2 & $2.44 \times 10^{7}$ \\
\hline P56480 & ATP synthase subunit beta, mitochond & drial & 7.64 & 6.05 & 1 & $1.39 \times 10^{7}$ & 56.3 & 0 (hypoxia only) \\
\hline Q03265 & ATP synthase subunit alpha, mitochon & drial & 8.02 & 5.97 & 1 & $9.67 \times 10^{6}$ & 59.7 & $1.25 \times 10^{7}$ \\
\hline P63038 & $60 \mathrm{kDa}$ heat shock protein, mitochond & drial & 5.16 & 3.66 & 1 & $7.45 \times 10^{6}$ & 60.9 & 0 (hypoxia only) \\
\hline
\end{tabular}

${ }^{a}$ Hypoxic regions were identified by IHC staining, and LCM was performed to isolate tissues from hypoxic regions. The captured tissues were then digested in solution and submitted to LC-MS/MS analysis. Proteome Discoverer 1.3 was used for analysis. Proteins that were found to be associated with hypoxia are displayed. An extensive list of identified proteins can be found in the Supporting Information.

hypoxic regions of the tumor tissue sections. Additionally, galectin-1, a well-known hypoxia-induced protein, was also among the top 20 proteins in the list. Some proteins that have unreported hypoxic regulation but whose functions are associated with hypoxia response were also detected in either hypoxic regions or both hypoxic and normoxic regions. These include $60 \mathrm{kDa}$ heat shock protein, which was found to be exclusively present in hypoxic regions.

\section{Comparison between in Vitro and in Situ LC-MS/MS Data Set}

Data obtained from in vitro quantitative proteomic LC-MS/ MS analyses were compared with those obtained from LCMLC-MS/MS analyses (see Supporting Information Table S4). Among the top 131 proteins found to be up- or down-regulated under hypoxic conditions following SILAC LC-MS/MS, 38 proteins were identified and found to be present in hypoxic or normoxic regions following LCM-LC-MS/MS. Twenty-six proteins were found to be common between up-regulated proteins under hypoxic conditions from SILAC LC-MS/MS studies and proteins identified in LCM hypoxic regions. These include proteins such as phosphoglycerate kinase 1, galectin, and annexin. Figure 4 shows a diagram to visualize the common and distinct proteins in all analysis data sets.

\section{Localization of Hypoxia in 4T1 Primary Tumor Tissue Sections Using MALDI-MS Imaging}

FFPE pimo-treated 4T1 tumor tissue sections were subjected to IHC staining to locate hypoxic regions. Figure 5A shows the localization of hypoxic regions within a whole FFPE 4T1 tumor tissue section by IHC staining for pimo. Serial sections were then subjected to MALDI-MSI and on-tissue MS/MS analyses to obtain molecular information and protein identification. Figure $5 \mathrm{~B}$ shows a resulting average MALDI mass spectrum acquired from one pixel across the tissue section. Direct MS/MS results were subjected to MASCOT search, and this allowed the identification of 15 proteins directly from the tissue section. This enabled the identification of mostly abundant proteins including hemoglobin, actin, histone, and vimentin. Here combining both LC-MS/MS and MALDI-MSI results enabled the localization and identification of proteins shown in Figure 5C. The protein localizations displayed in Figure 5C were accepted only if at least two peptides arising from the same protein (based on quantitative LC-MS/MS analyses from both in vitro and LCM samples) showed a similar 


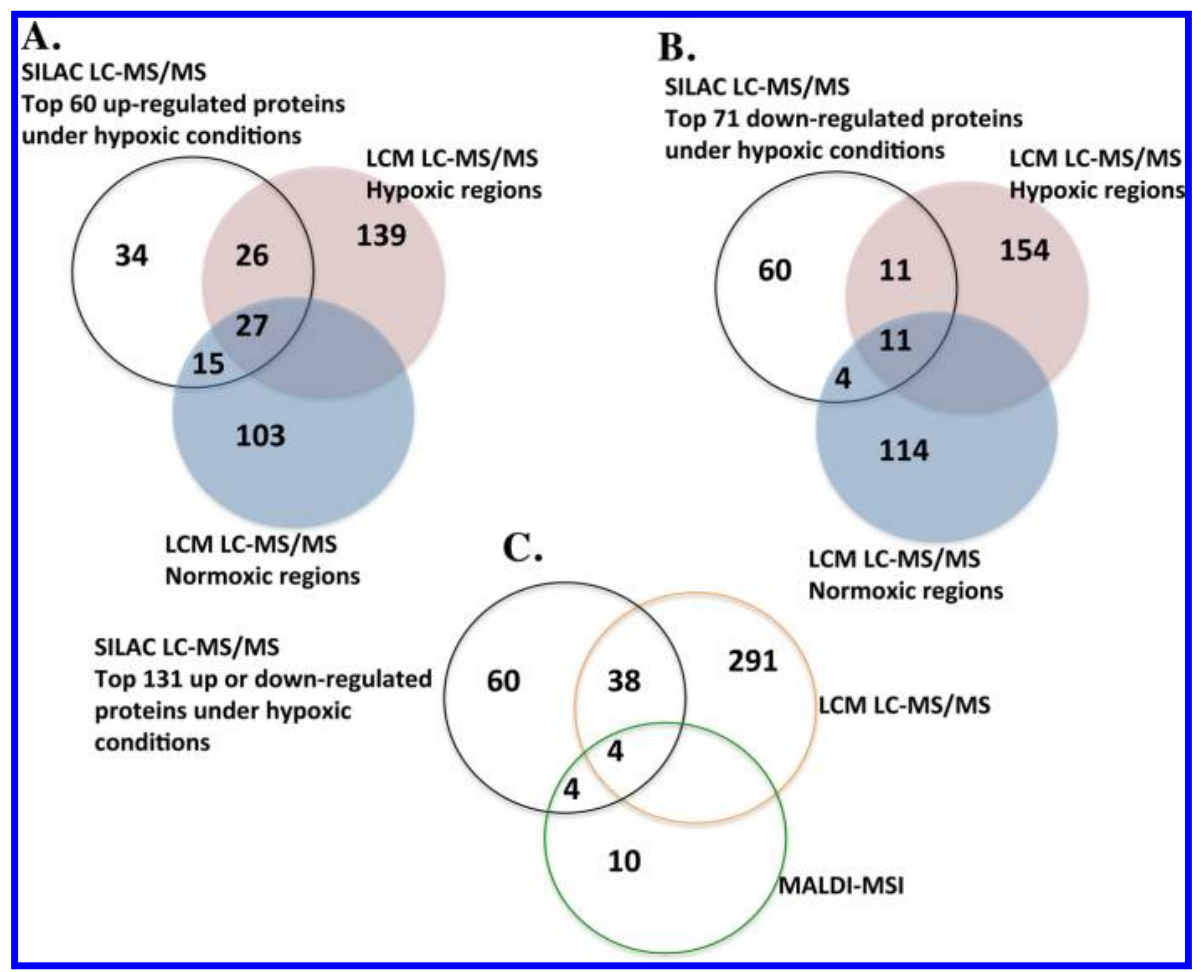

Figure 4. Venn diagram of the number of common and distinct proteins identified with SILAC LC-MS/MS, LCM LC-MS/MS, and MALDI-MSI. (A) Comparison between the observed top 60 up-regulated proteins following SILAC LC-MS/MS analysis with those identified after LCM LCMS/MS analysis of 4T1 pimo-treated tumor tissue sections. (B) Comparison between the observed top 71 down-regulated proteins following SILAC LC-MS/MS analysis with those identified after LCM LC-MS/MS analysis of 4T1 pimo-treated tumor tissue sections. (C) Comparison between the number of successfully identified proteins using SILAC LC-MS/MS, LCM LC-MS/MS and MALDI-MSI analyses.

distribution across the tissue section. Such approaches have already been demonstrated and used by Chaurand et al. ${ }^{20}$ Using both MALDI-MSI and quantitative LC-MS/MS, we confirmed the distribution of proteins identified within 4T1 tumor tissue sections. Figure 5C displays the localization of peptides arising from several proteins, including classic hypoxia markers phosphoglycerate kinase- 1 and galectin. When comparing the MALDI-MS images with the immunostaining for regions of hypoxia, we noted that the displayed peptides were mainly localized to and relatively abundant in hypoxic regions. Also, the distribution of peptides arising from hemoglobin alpha- and beta-chain $(m / z 1529$ and 1274$)$ showed the presence of blood supply in normoxic regions adjacent to the hypoxic regions. The results obtained by MALDI-MSI analyses enabled localization of hypoxia-regulated proteins directly in tumor tissue sections. These results also highlight the advantage of MALDIMSI over traditional IHC methods in localization of the protein of interest, as IHC allows only one target to be detected per sample, whereas MALDI-MSI allows a multitude of targets to be detected in a single experiment.

Validation of Hypoxia-Associated Proteins Using Statistical Analysis of MALDI-MSI Data

Here we developed a computational pipeline to match proteins identified with MALDI-MSI to the hypoxic/normoxic areas identified through IHC staining. Query proteins fibronectin, galectin, hemoglobin, heat shock protein 27, and phosphoglycerate kinase were tested for association with hypoxia, that is, whether they have significantly higher or lower expression in hypoxic tissue compared with normoxic tissue. In Figure 6A,B, boxplots and density distributions of peak intensities of phosphoglycerate peptides $(1083 \mathrm{~m} / z)$ stemming from hypoxic (red) and normoxic (blue) areas of the section are shown. Using the Student's $t$ test, the hypothesis of equal mean, that is, same level of average expression in hypoxia and normoxia, can be rejected in favor of the alternative hypothesis with a significant $p$ value of $1.3 \times 10^{-35}$. As shown in Table 3 , all tested proteins except hemoglobin A exhibit a statistically significant different $(p<0.01)$ mean expression between normoxic and hypoxic tissue.

\section{Protein Network Analysis}

We developed an algorithm whereby all proteins consistently detected in all repeats of the in vitro SILAC system were analyzed using the STRING database. We extracted the associations of all MS-detected proteins and visualized the resulting network using Cytoscape, with proteins that were significantly up- or down-regulated (defined as at least 1.4-fold change) highlighted as either red or blue nodes, respectively, and those that exhibited no change in white (Figure 7A,B). This enabled us to visualize the connections between the detected proteins and build up an interaction network even though not all proteins were significantly altered, thereby providing a 'snapshot' of the true state of the protein networks within the cells at the time of sample collection. As can be seen from Figure 7A, the detected proteins in 4T1 samples formed extensive complex networks. There is one major network containing several interconnected hubs. This observation is as expected because proteins in cells generally interact with each other for many purposes. Additionally, proteins with only one connection were also detected.

In addition to visualization using Cytoscape, an algorithm was developed to further interrogate the individual proteins with significant changes between hypoxia and normoxia, by 


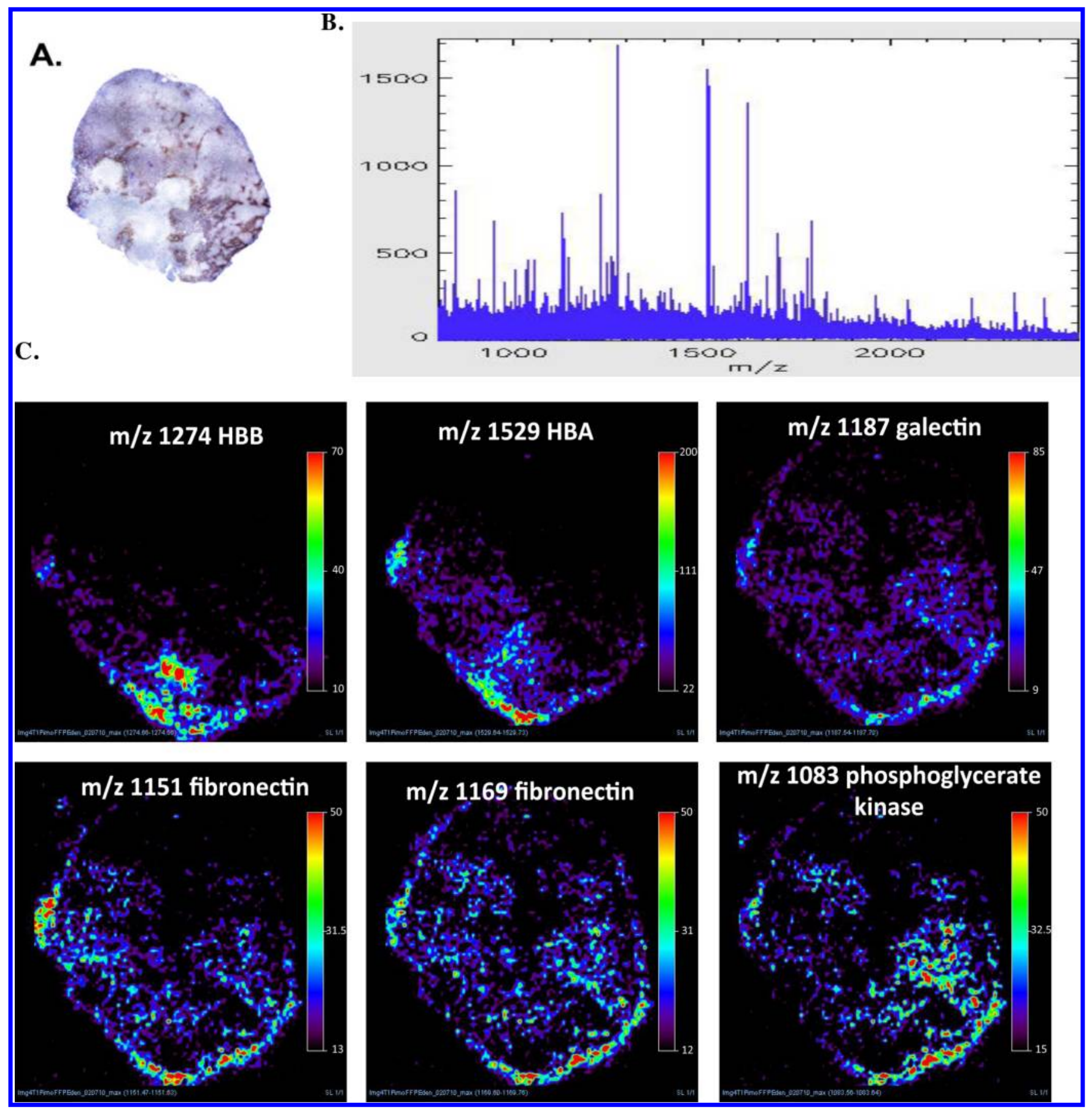

Figure 5. Immunohistochemistry staining of a 4T1 FFPE tumor tissue section and MALDI-MSI analysis. (A) IHC staining of pimo localization within a FFPE 4T1 pimo-treated tumor tissue section. Serial tissue sections were then subjected to MALDI-MSI analysis. (B) MALDI-mass spectrum acquired from a FFPE 4T1 pimo-treated tumor tissue section. (C) MALDI-MS images of peptides within the tissue sections. The localizations of galectin, fibronectin, and phosphoglycerate kinase within pimo-treated tumor tissue sections indicate that hypoxic regions are displayed. This was found to be in good agreement with the IHC staining of pimo within the tumor tissue sections. HBB and HBA are also shown to indicate the presence of blood vessel in the tumor.

identifying significantly altered proteins that are neighbors with another significantly altered protein (Table 4). The Table was subdivided into four parts according to the detected hypoxia levels assigned to each protein, with "up" (i.e., fold-change of heavy label (normoxia) /light label (hypoxia) < 0.714) meaning the protein was up-regulated in hypoxia, and "down" (i.e., foldchange of heavy label (normoxia)/light label (hypoxia) > 1.4) meaning the protein was down-regulated in hypoxia: up-up, up-down, down-up, and down-down. This allows us to extrapolate information on potential protein regulators from the extensive network. For example, the protein Tpr was detected to be down-regulated in hypoxia, and its neighboring proteins were also down-regulated. Interestingly, it has been suggested that Tpr regulated the export of mRNAs, ${ }^{21}$ indicating a regulatory role of Tpr in protein translation. Additionally, we can pinpoint proteins that are regulated by the same upstream signals; for example, in 4T1 cells, glyceraldehyde-3-phosphate dehydrogenase, enolase-1, lactate dehydrogenase-A, phophoglycerate 


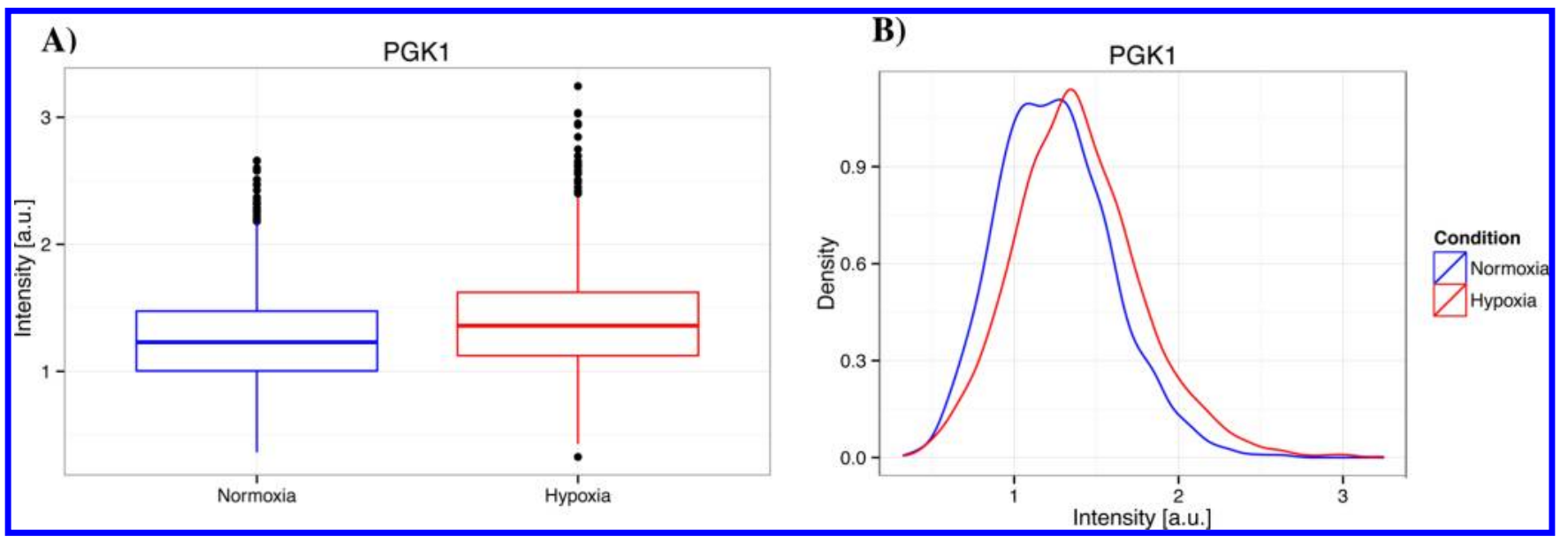

Figure 6. Distribution of peak intensities of phosphoglycerate kinase in normoxic (blue) and hypoxic (red) tissue. (A) Boxplots and (B) density distributions of peptide intensities show that average expression of phosphoglycerate kinase is significantly higher in hypoxic tissue compared with normal tissue (Student's $t$ test, $p$ value $<0.01$ ). High variance of peptide intensities in both tissue types is likely due to the coarse grained acquisition of MALDI MS spectra $(80 \mu \mathrm{m})$, spanning multiple cells.

Table 3. Query Proteins Tested for Equal Mean of Expression in Hypoxic and Normoxic Tissue, Respectively ${ }^{a}$

\begin{tabular}{lcc}
\multicolumn{1}{c}{ protein } & matched peptide $\mathrm{m} / z$ & $t$ test $[P$ value $]$ \\
fibronectin & 1151.6 & $2.80 \times 10^{-3}$ \\
& 1169.7 & $4.80 \times 10^{-9}$ \\
galectin & 1187.5 & $6.50 \times 10^{-8}$ \\
hemoglobin A & 1529.7 & $2.20 \times 10^{-3}$ \\
hemoglobin B & 1274.7 & $4.50 \times 10^{-1}$ \\
heat Shock protein 27 & 987.6098 & $5.10 \times 10^{-19}$ \\
phosphoglycerate kinase & 1083.6044 & $1.30 \times 10^{-35}$
\end{tabular}

${ }^{a}$ Query proteins were in silico digested and the observed peptide masses were compared to the theoretical values. Populations of peak intensities from normoxic and hypoxic tissue were tested for equal mean using a two sample Student's t-test. All tested proteins, except hemogolobin $\mathrm{B}$, showed a significant $(p<0.01)$ difference of mean expression between both tissue types.

kinase-1, and aldolase-A all neighbor triosphophate isomerase-1. These are all glycolysis pathway proteins and are known to be up-regulated in hypoxia. ${ }^{22}$ We can also use this tool to identify potential protein-protein interactions within the system. For example, RanBP2 neighbors Nup214; it has been previously established that RanBP2 attaches to the nuclear pore complex via association with Nup214. ${ }^{23}$

\section{DISCUSSION}

Hypoxia, which occurs in most solid tumors, is clinically correlated with metastatic progression, treatment failure, and decreased patient survival. Therefore, the identification of hypoxia-regulated proteins that drive metastasis as well as understanding the molecular networks that are associated with hypoxia could lead to the identification of novel therapeutic strategies.

In the study reported here, several approaches have been combined to identify in vitro and in vivo protein changes in normoxic versus hypoxic conditions. LC-MS/MS analysis of SILAC-labeled breast cancer cells has allowed a relative quantitative comparison of protein changes observed in both normoxia and hypoxia. 131 proteins found to be hypoxiamodulated were identified. However, the quantification methodology can be improved by using in-solution tryptic digest as opposed to the in-gel digestion used in this study to minimize potential keratin contamination and thus maximize the sensitivity in protein detection/discovery. ${ }^{24}$ In addition, the presence of Coomassie Blue in the SDS-PAGE gel may interfere with digestion, again suggesting that in-solution digestion may be a better option in studies such as ours. MALDI-MSI is a fast growing technique with the ability to localize proteins directly within tissue sections with no need for predefined targets. Hence, it is a powerful tool for translational research strategies. Here the use of MALDI-MSI has allowed the distribution of several proteins within breast tumor tissue sections to be visualized as well as hypoxic and normoxic regions to be distinguished. Such information can then be correlated with histology and IHC studies to have greater insight into the association between tumor morphology and molecular biology. The distribution of these peptides as well as their relative intensities across the tumor tissue sections were in good agreement with the quantitative analysis. Importantly, the statistical analysis of the selected MALDI-MSI detected peptides with IHC staining revealed strong verification of the presence of these peptides in the hypoxic areas. These techniques, when combined together, suggest the great potential of MALDI-MSI to be used as a blind-search tool to discover novel proteins associated with tissue microenvironments. The direct identification of proteins within tissue sections has also been demonstrated in several studies. ${ }^{7 b, 9 a, c}$ Here we used LCM to isolate the regions of interest within the tumor tissue sections prior to LC-MS/MS analyses for protein identification. Using LCM prior to LC-MS/MS analysis enabled the identification of 118 and 164 proteins in situ in the normoxic and hypoxic areas of the 4T1 tumor tissue sections, respectively.

The combination of techniques used in this study, including LC-MS/MS SILAC-labeled cell proteomics and LCM LC-MS/MS on tumor tissue sections, allowed the identification and quantification of a large number of proteins within mammary tumors as well as distinguished hypoxic and normoxic regions within the tumor environment. Combining these technologies with MALDI-MSI brings specificity to the methodology used here and also enables the localization of several proteins within the tissue section, including lowabundant proteins as well as targeted proteins, that is, 


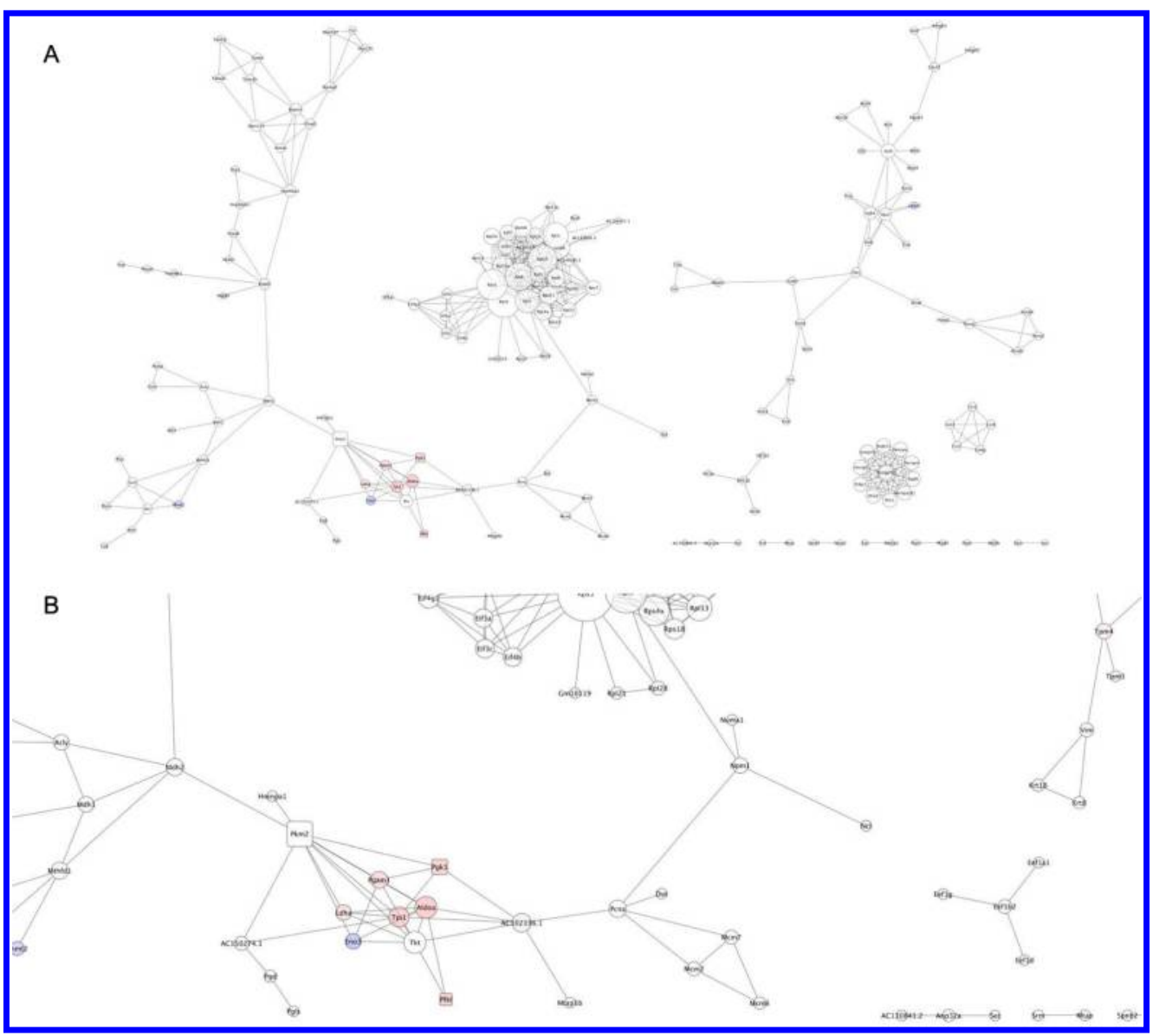

Figure 7. Cytoscape visualization of in vitro protein network in 4T1 mammary cancer cells. Results from in vitro MS study on 4T1 hypoxic- and normoxic-treated cells were run through algorithms to generate a view of the connections between MS-detected proteins. Red nodes denote proteins significantly up-regulated in hypoxia, blue nodes denote proteins significantly down-regulated in hypoxia, and white nodes denote proteins with no significant changes between hypoxia and normoxia. Sizes of nodes correspond to degree of connection, and kinases were displayed as squares. (A) Zoom-in of the major network with interconnected hubs is displayed. (B) Zoom-in of one of the individual subnetworks detected. Single-connection proteins are also detected.

hypoxia-related proteins. The combination of LCM-LC-MS/MS with MALDI-MSI enabled the identification and localization of several proteins within the tumor tissue sections. Because no separation step is performed prior to MALDI-MSI analysis, this results more often in a crude mixture of peptides with other biomolecular species including lipids, small molecules, as well as protein modifications being present. Hence, on-tissue MALDIMS/MS analyses lead to poor results when compared with conventional LC-MS/MS due to sample complexity. However, as demonstrated here, using both data sets from LCM-LCMS/MS and MALDI-MSI allowed us, on one hand, to obtain the proteomic information contained in the sample; on the other hand, we were able to associate this information with the spatial localization within the tissue section. This can be visualized in Figure 4, which shows the combined results from all analyses including SILAC LC-MS/MS, LCM-MS/MS, and MALDI-MSI data. The discrepancies observed between these in situ studies (i.e., LCM LC-MS/MS and MALDI-MSI) may be explained by the different preservation techniques of tumor sections, as frozen tissues were used for LCM LC-MS/MS, whereas we could only perform MALDI-MSI on FFPEpreserved tissues successfully (data not shown). The freezethawing of tumor sections may have caused degradation of proteins. In addition, the use of a Denator system on the FFPE tissue sections used for MALDI-MSI provided further stabilization of proteins. Furthermore, in vitro and in vivo environment can elicit different responses in cancer cells; the discrepancies between our in vitro SILAC results and in situ LCM LC-MS/MS results highlight this issue.

Here MALDI-MSI analyses of on-tissue digested proteins generated large data sets, which demonstrated differences between peptide profiles when comparing hypoxic versus normoxic regions, hence reflecting the complexity and heterogeneity of the molecular nature of the disease. There is thus a huge need for bioinformatic studies to create tools that allow network building and results management. We have achieved this in our study by developing a tool to visualize the interaction network between in vitro MS-detected proteins. The algorithm demonstrated here allowed for visualization of large data sets without bias but still retained information that might be important. The algorithm applied stringent filters for identification of the proteins but also presented identified proteins whose expressions were not 'significantly' changed (i.e., 1.4 fold change in this study). This allowed a true view of the network status of the various proteins at the time of sample collection and may reveal a 'hub' of proteins that were interconnected, which were significantly changed in expression by hypoxia. For example, the protein network highlighted in Figure $7 \mathrm{~B}$ is involved in the glycolytic pathway. This is not surprising because hypoxia is known to induce a shift from the 
Table 4. List of in Vitro MS-Detected Proteins with Significant Changes between Hypoxia and Normoxia with Neighbors of Other Significantly Altered Protein in 4T1 Mammary Cancer Cells ${ }^{a}$

\begin{tabular}{|c|c|c|c|c|c|c|c|c|c|c|c|}
\hline enquiry protein & score & neighbor 1 & score 1 & neighbor 2 & score 2 & neighbor 3 & score 3 & neighbor 4 & score 4 & neighbor 5 & score 5 \\
\hline Nup214 & down & Ranbp2 & down & Tpr & down & & & & & & \\
\hline Vim & down & Krt18 & down & & & & & & & & \\
\hline Hspd1 & down & Hspa9 & down & & & & & & & & \\
\hline Krt18 & down & Vim & down & & & & & & & & \\
\hline Ranbp2 & down & Nup214 & down & Tpr & down & & & & & & \\
\hline Tpr & down & Nup214 & down & Ranbp2 & down & & & & & & \\
\hline Hspa9 & down & Hspd1 & down & & & & & & & & \\
\hline Vim & down & Tpm4 & up & & & & & & & & \\
\hline Eno3 & down & Pgam1 & up & Aldoa & up & & & & & & \\
\hline Tpm4 & up & Vim & down & & & & & & & & \\
\hline Aldoa & up & Eno3 & down & & & & & & & & \\
\hline Pgam1 & up & Eno3 & down & & & & & & & & \\
\hline Gapdh & up & Tpil & up & & & & & & & & \\
\hline Pfkl & up & Aldoa & up & & & & & & & & \\
\hline Ldha & up & Tpil & up & Aldoa & up & & & & & & \\
\hline Aldoa & up & Gapdh & up & Ldha & up & Pgam1 & up & Tpil & up & Pfkl & up \\
\hline Pgk1 & up & Gapdh & up & Pgam 1 & up & Tpil & up & & & & \\
\hline Tpil & up & Eno1 & up & Gapdh & up & Ldha & up & Pgk1 & up & Aldoa & up \\
\hline Enol & up & Tpil & up & & & & & & & & \\
\hline Pgam 1 & up & Pgk1 & up & Aldoa & up & & & & & & \\
\hline Gapdh & up & Pgk1 & up & Tpil & up & Aldoa & up & & & & \\
\hline
\end{tabular}

${ }^{a}$ Results from in vitro MS study on $4 \mathrm{~T} 1$ hypoxic- and normoxic-treated cells were run through algorithms to extrapolate significantly changed proteins that are neighbors with other significantly altered protein(s). Proteins were scored 'up' or 'down', indicating 'significant down-regulation in hypoxia' and 'significant up-regulation in hypoxia', respectively.

normal oxidative phosphorylation that occurs in the mitochondria to the so-called 'anaerobic' glycolysis due to the limited amount of oxygen available to the cells. As such, these glycolytic enzymes presented in the Cytoscape network may be good therapeutic targets against hypoxia-mediated breast cancer progression. In addition to revealing 'hubs', the algorithm also presented proteins that may interact or regulate with the significantly regulated protein, as determined from the STRING score. This presentation of protein networks allows for identification of new potential drug targets; for example, if a protein identified is deemed 'undruggable' due to various reasons, by looking at the connections around it using the network, we could identify an interactor, upstream regulator or downstream effector to target instead. As such, the algorithm demonstrated here can be of immense value for handling large data sets.

HIF $1 \alpha$, arguably the most robust hypoxia-regulated protein, was not present in the results here. However, this is not due to the lack of robustness of our experimental approaches but rather due to the transient nature of the protein. An alternative approach to assessing hypoxia is the detection of more stable known hypoxia-regulated proteins.

The detection of well-characterized hypoxia-regulated proteins such as galectin- $1,{ }^{25}$ alpha-enolase, ${ }^{26}$ and $78 \mathrm{kDa}$ glucose-regulated protein (GRP78) ${ }^{27}$ in the samples is indicative of the robustness of our detection system. These proteins were also detected in our in vitro SILAC system, which further confirms the validity of our study. In addition, further well-known hypoxia-induced proteins such as glyceraldehyde-3phosphate dehydrogenase (GAPDH, or G3P in mouse), L-lactate dehydrogenase (LDHA), phosphoglycerate kinase 1 (PGK1), aldolase A (ALDOA), and alpha-enolase (ENOA) were also present in the list of significantly hypoxia up-regulated proteins in the in vitro SILAC studies, highlighting the robustness of our hypoxic system. $^{26,28}$

To date, there has been only one other study investigating an in situ molecular profile of the hypoxic tumor microenvironment. Marotta and colleagues also used the LCM technique to isolate hypoxic tissues, as we have done in this study, although they focused on the mRNA levels as opposed to our proteomics approach. ${ }^{29}$ Nevertheless, one could expect to see some overlap between their mRNA profile and our proteomics profile. Interestingly, none of the proteins encoded by the top 20 up/down-regulated mRNA changes observed by Marotta et al. (2011) were detected in our in situ tumor sections. This could be attributed to the fact that mRNA transcription does not necessarily lead to translation and also to the differences in cancer models and species that were used in the two studies: Marotta et al. studied gliomas in rats, whereas we studied mammary tumors in mice.

There appears to be more overlap between our individual in vitro data sets (see the table in the Supporting Information) and the mRNA data produced by Marotta and colleagues. This could be due to the more homogeneous nature of in vitro systems compared with in vivo systems. However, if we look at the combined results from all three SILAC in vitro repeats, Marotta et al. did not detect any of the proteins consistently regulated by hypoxia in our study at the mRNA level. This is likely due to the same reasons previously listed: the fact that mRNA levels do not necessarily reflect protein dynamics, in addition to the differences in cell lines and hypoxia treatments. Additionally, the in-gel digestion for extraction of in vitro SILAC samples may not have been completely efficient across the three experiments, and the overlap between the repeats may be improved by other sample preparation methods, such as insolution digestion. 
Of the proteins that were identified in both in vitro SILAC and in situ LCM mass spectrometry analyses, we have identified a novel hypoxia-regulated protein tropomysin alpha-4 chain (TPM4), which was found to be significantly up-regulated in vitro in hypoxic 4T1 cells and was also detected in the hypoxic regions of the 4T1 tumor samples. TPM4 is involved in regulating actin filaments of cells, and while there has been no previous report suggesting a link between TPM4 and hypoxia, previous immunohistochemical analysis on lymph node metastases of breast cancer patients showed a significant association of TPM4 with metastasis. ${ }^{30}$ Hypoxia is known to enhance metastasis; ${ }^{31}$ as such, the hypoxia-induced TPM4 reported here may be involved in modifying the actin cytoskeleton and mobilizing cancer cells to metastasize. It would be interesting to validate this hypothesis by knocking down TPM4 and observing the effects on hypoxiainduced invasion of 4T1 cells in a future study. Nonetheless, here we have demonstrated that TPM4 may be a novel hypoxic marker for breast cancer cells.

Interestingly, one of the most down-regulated proteins in the in vitro hypoxic $4 \mathrm{~T} 1$ cells was the histone $\mathrm{H} 2 \mathrm{~B}$ type 1 protein. Family members of this $\mathrm{H} 2 \mathrm{~B}$ type 1 protein were also detected in our in situ LCM samples, alongside with other histone proteins $(\mathrm{H} 3, \mathrm{H} 4)$. Similar to TPM4, there has been no study linking the expression of $\mathrm{H} 2 \mathrm{~B}$ type 1 protein to hypoxia, suggesting that this group of proteins may potentially be novel hypoxic-regulated proteins. Histones are responsible for the packaging and structural arrangement of DNA into chromatins and undergo posttranslational modifications to alter their interactions with DNA and nuclear proteins. These modifications are also involved in regulating the chromatin structures and thus transcriptional control of the DNA within (known as epigenetic control). If histones were lost, then one could imagine the DNA to become disorganized, and the control over transcription of said DNA would be lost, thereby introducing genomic instability. This presents a very interesting new mechanism to which hypoxia may induce genomic instability.

\section{CONCLUSIONS}

We have reported here the first comprehensive study on hypoxia-regulated protein changes. We utilized several MS-based approaches to identify hypoxia-regulated proteins in mammary cancer cells in vitro and in situ and visualized these proteins within tumor sections using MALDI-MSI. We further provided a pipeline to which sections that underwent MALDIMSI could be directly compared with pimonidazole-stained IHC sections and identified peptides that were significantly localized in hypoxic areas. This presents an exciting prospect that with further optimization these techniques could provide a powerful tool to identify specific proteins involved in the metastatic process. We have also demonstrated the use of bioinformatics to aid the analysis of an extensive proteomics network. The need to develop bioinformatics tools can be highlighted by the complex results generated by mass-spectrometry methods, as demonstrated by our results here, wherein vitro and in situ results may not be in concordance. A computational approach to bringing the results together is essential in dealing with large data sets generated by MS techniques.

\section{ASSOCIATED CONTENT}

\section{S Supporting Information}

Spectrum peak identification and alignment, query protein to peak cluster matching, spectrum label assignment, and statistical assessment of association to hypoxia. An example of co-registered IHC stained image with MALDI-MS image. List of identified up- or down-regulated proteins under hypoxic conditions. Identified proteins from tissues isolated from in vivo hypoxic regions of 4T1 primary tumour sections after LCMLC-MS/MS. Identified proteins from tissues isolated from in vivo normoxic regions of $4 \mathrm{~T} 1$ primary tumour sections after LCM-LC-MS/MS. Comparison between all datasets. This material is available free of charge via the Internet at http:// pubs.acs.org.

\section{AUTHOR INFORMATION}

\section{Corresponding Author}

*E-mail: janine.erler@bric.ku.dk.

\section{Present Addresses}

"J.C.: Biotech Research and Innovation Centre (BRIC), University of Copenhagen, Ole Maaløes Vej 5, Copenhagen 2200, Denmark.

${ }^{\#}$ M.-C.D.: Novartis Pharma, Biologics Process Research and Development - Analytical Development. Klybeckstrasse 141, $\mathrm{CH}-4057$ Basel, Switzerland.

$\nabla_{\text {F.S.: }}$ ETH Zurich, D-BSSE. Computational Biology Group (CBG), office 8.42, Mattenstrasse 26, CH-4058 Basel, Switzerland.

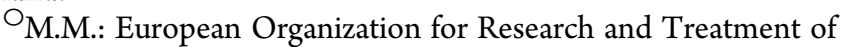
Cancer (EORTC), Avenue Mounier, 83/11, 1200 Brussels, Belgium.

Author Contributions

M.-C.D. and J.C. contributed equally.

Notes

The authors declare no competing financial interest.

\section{ACKNOWLEDGMENTS}

This work was supported by funding from the Association for International Cancer Research (M.-C.D.), the Breast Cancer Campaign (J.C.), Institute of Cancer Research (F.S., A.H., J.S., H.E.B., C.J., J.T.E.), MITRAC (M.M.), and CRUK (J.T.E.). We also give special thanks to $A B$ Sciex and Leica for the kind loan of their instruments to complete these studies.

\section{ABBREVIATIONS}

MALDI, matrix-assisted laser desorption/ionization; MSI, mass spectrometry imaging; LCM, laser capture microdissection; LC, liquid chromatography; MS, mass spectrometry; SILAC, stable isotope labeling with amino acids in cell culture; FFPE, formalin-fixed, paraffin-embedded; ITO, indium-tin-oxide

\section{REFERENCES}

(1) Carroll, V. A.; Ashcroft, M. Targeting the molecular basis for tumour hypoxia. Expert Rev. Mol. Med. 2005, 7 (6), 1-16.

(2) Vaupel, P.; Mayer, A. Hypoxia in cancer: significance and impact on clinical outcome. Cancer Metastasis Rev. 2007, 26 (2), 225-239.

(3) (a) Harris, A. L. Hypoxia-a key regulatory factor in tumour growth. Nat. Rev. Cancer 2002, 2 (1), 38-47. (b) Vaupel, P.; Hockel, M. Hypoxia in cervical cancer: pathogenesis, characterization, and biological/clinical consequences. Zentralbl. Gynakol. 2001, 123 (4), 192-197. (c) Vaupel, P.; Kelleher, D. K.; Hockel, M. Oxygen status of malignant tumors: pathogenesis of hypoxia and significance for tumor therapy. Semin. Oncol. 2001, 28 (2 Suppl 8), 29-35.

(4) (a) Chaudary, N.; Hill, R. P. Hypoxia and metastasis. Clin. Cancer Res. 2007, 13 (7), 1947-1949. (b) Erler, J. T.; Weaver, V. M. Threedimensional context regulation of metastasis. Clin. Exp. Metastasis 
2009, 26 (1), 35-49. (c) Finger, E. C.; Giaccia, A. J. Hypoxia, inflammation, and the tumor microenvironment in metastatic disease. Cancer Metastasis Rev. 2010, 29 (2), 285-293. (d) Overgaard, J.; Horsman, M. R. Modification of Hypoxia-Induced Radioresistance in Tumors by the Use of Oxygen and Sensitizers. Semin. Radiat. Oncol. 1996, 6 (1), 10-21. (e) Erler, J. T.; Giaccia, A. J. Lysyl oxidase mediates hypoxic control of metastasis. Cancer Res. 2006, 66 (21), $10238-10241$.

(5) Caprioli, R. M.; Farmer, T. B.; Gile, J. Molecular imaging of biological samples: localization of peptides and proteins using MALDITOF MS. Anal. Chem. 1997, 69 (23), 4751-4760.

(6) Trim, P. J.; Francese, S.; Clench, M. R. Imaging mass spectrometry for the assessment of drugs and metabolites in tissue. Bioanalysis 2009, 1 (2), 309-319.

(7) (a) Chaurand, P.; Stoeckli, M.; Caprioli, R. M. Direct profiling of proteins in biological tissue sections by MALDI mass spectrometry. Anal. Chem. 1999, 71 (23), 5263-5270. (b) Heeren, R. M.; Smith, D. F.; Stauber, J.; Kukrer-Kaletas, B.; MacAleese, L. Imaging mass spectrometry: hype or hope? J. Am. Soc. Mass Spectrom. 2009, 20 (6), 1006-14. (c) Stoeckli, M.; Staab, D.; Schweitzer, A.; Gardiner, J.; Seebach, D. Imaging of a beta-peptide distribution in whole-body mice sections by MALDI mass spectrometry. J. Am. Soc. Mass Spectrom. 2007, 18 (11), 1921-1924.

(8) (a) Rauser, S.; Deininger, S. O.; Suckau, D.; Hofler, H.; Walch, A. Approaching MALDI molecular imaging for clinical proteomic research: current state and fields of application. Expert Rev. Proteomics 2010, 7 (6), 927-941. (b) Rauser, S.; Marquardt, C.; Balluff, B.; Deininger, S. O.; Albers, C.; Belau, E.; Hartmer, R; Suckau, D.; Specht, K.; Ebert, M. P.; Schmitt, M.; Aubele, M.; Hofler, H.; Walch, A. Classification of HER2 receptor status in breast cancer tissues by MALDI imaging mass spectrometry. J. Proteome Res. 2010, 9 (4), 1854-1863. (c) Wisztorski, M.; Lemaire, R.; Stauber, J.; Menguelet, S. A.; Croix, D.; Mathe, O. J.; Day, R.; Salzet, M.; Fournier, I. New developments in MALDI imaging for pathology proteomic studies. Curr. Pharm. Des. 2007, 13 (32), 3317-3324.

(9) (a) Djidja, M. C.; Claude, E.; Snel, M. F.; Francese, S.; Scriven, P.; Carolan, V.; Clench, M. R. Novel molecular tumour classification using MALDI-mass spectrometry imaging of tissue micro-array. Anal. Bioanal. Chem. 2010, 397 (2), 587-601. (b) Groseclose, M. R; Massion, P. P.; Chaurand, P.; Caprioli, R. M. High-throughput proteomic analysis of formalin-fixed paraffin-embedded tissue microarrays using MALDI imaging mass spectrometry. Proteomics 2008, 8 (18), 3715-3724. (c) Lemaire, R.; Desmons, A.; Tabet, J. C.; Day, R.; Salzet, M.; Fournier, I. Direct analysis and MALDI imaging of formalin-fixed, paraffin-embedded tissue sections. J. Proteome Res. 2007, 6 (4), 1295-1305.

(10) Aslakson, C. J.; Miller, F. R. Selective events in the metastatic process defined by analysis of the sequential dissemination of subpopulations of a mouse mammary tumor. Cancer Res. 1992, 52 (6), 1399-1405.

(11) Jorgensen, C.; Sherman, A.; Chen, G. I.; Pasculescu, A.; Poliakov, A.; Hsiung, M.; Larsen, B.; Wilkinson, D. G.; Linding, R.; Pawson, T. Cell-specific information processing in segregating populations of Eph receptor ephrin-expressing cells. Science 2009, 326 (5959), 1502-1509.

(12) United Kingdom Co-ordinating Committee on Cancer Research (UKCCCR) Guidelines for the Welfare of Animals in Experimental Neoplasia (2nd Edition). Br. J. Cancer 1998, 77 (1), 1-10.

(13) Djidja, M. C.; Claude, E.; Snel, M. F.; Scriven, P.; Francese, S.; Carolan, V.; Clench, M. R. MALDI-ion mobility separation-mass spectrometry imaging of glucose-regulated protein $78 \mathrm{kDa}$ (Grp78) in human formalin-fixed, paraffin-embedded pancreatic adenocarcinoma tissue sections. J. Proteome Res. 2009, 8 (10), 4876-4884.

(14) Lemaire, R.; Tabet, J. C.; Ducoroy, P.; Hendra, J. B.; Salzet, M.; Fournier, I. Solid ionic matrixes for direct tissue analysis and MALDI imaging. Anal. Chem. 2006, 78 (3), 809-819.

(15) Szklarczyk, D.; Franceschini, A.; Kuhn, M.; Simonovic, M.; Roth, A.; Minguez, P.; Doerks, T.; Stark, M.; Muller, J.; Bork, P.; Jensen, L. J.; von Mering, C. The STRING database in 2011: functional interaction networks of proteins, globally integrated and scored. Nucleic Acids Res. 2011, 39 (Database issue), D561-D568.

(16) Smoot, M. E.; Ono, K.; Ruscheinski, J.; Wang, P. L.; Ideker, T. Cytoscape 2.8: new features for data integration and network visualization. Bioinformatics 2011, 27 (3), 431-432.

(17) Bos, R.; Zhong, H.; Hanrahan, C. F.; Mommers, E. C.; Semenza, G. L.; Pinedo, H. M.; Abeloff, M. D.; Simons, J. W.; van Diest, P. J.; van der Wall, E. Levels of hypoxia-inducible factor-1 alpha during breast carcinogenesis. J. Natl. Cancer Inst. 2001, 93 (4), 309-314.

(18) Erler, J. T.; Bennewith, K. L.; Cox, T. R.; Lang, G.; Bird, D.; Koong, A.; Le, Q. T.; Giaccia, A. J. Hypoxia-induced lysyl oxidase is a critical mediator of bone marrow cell recruitment to form the premetastatic niche. Cancer Cell 2009, 15 (1), 35-44.

(19) Raleigh, J. A.; Miller, G. G.; Franko, A. J.; Koch, C. J.; Fuciarelli, A. F.; Kelly, D. A. Fluorescence immunohistochemical detection of hypoxic cells in spheroids and tumours. Br. J. Cancer 1987, 56 (4), 395-400.

(20) Chaurand, P.; Fouchecourt, S.; DaGue, B. B.; Xu, B. J.; Reyzer, M. L.; Orgebin-Crist, M. C.; Caprioli, R. M. Profiling and imaging proteins in the mouse epididymis by imaging mass spectrometry. Proteomics 2003, 3 (11), 2221-2239.

(21) Coyle, J. H.; Bor, Y. C.; Rekosh, D.; Hammarskjold, M. L. The Tpr protein regulates export of mRNAs with retained introns that traffic through the Nxf1 pathway. RNA 2011, 17 (7), 1344-1356.

(22) Robin, E. D.; Murphy, B. J.; Theodore, J. Coordinate regulation of glycolysis by hypoxia in mammalian cells. J. Cell. Physiol. 1984, 118 (3), 287-290.

(23) Bernad, R; van der Velde, H.; Fornerod, M.; Pickersgill, H. Nup358/RanBP2 attaches to the nuclear pore complex via association with Nup88 and Nup214/CAN and plays a supporting role in CRM1mediated nuclear protein export. Mol. Cell. Biol. 2004, 24 (6), 23732384.

(24) Bell, A. W.; Deutsch, E. W.; Au, C. E.; Kearney, R. E.; Beavis, R.; Sechi, S.; Nilsson, T.; Bergeron, J. J. A HUPO Test Sample Study Reveals Common Problems in Mass Spectrometry-Based Proteomics. Nat. Methods 2009, 6 (6), 423-430.

(25) Le, Q. T.; Shi, G.; Cao, H.; Nelson, D. W.; Wang, Y.; Chen, E. Y.; Zhao, S.; Kong, C.; Richardson, D.; O’Byrne, K. J.; Giaccia, A. J.; Koong, A. C. Galectin-1: a Link between Tumor Hypoxia and Tumor Immune Privilege. J. Clin. Oncol. 2005, 23 (35), 8932-8941.

(26) Semenza, G. L.; Jiang, B. H.; Leung, S. W.; Passantino, R.; Concordet, J. P.; Maire, P.; Giallongo, A. Hypoxia response elements in the aldolase $\mathrm{A}$, enolase 1 , and lactate dehydrogenase $\mathrm{A}$ gene promoters contain essential binding sites for hypoxia-inducible factor 1. J. Biol. Chem. 1996, 271 (51), 32529-32537.

(27) (a) Koong, A. C.; Auger, E. A.; Chen, E. Y.; Giaccia, A. J. The regulation of GRP78 and messenger RNA levels by hypoxia is modulated by protein kinase $\mathrm{C}$ activators and inhibitors. Radiat. Res. 1994, 138 (1 Suppl), S60-S63. (b) Song, M. S.; Park, Y. K.; Lee, J. H.; Park, K. Induction of glucose-regulated protein 78 by chronic hypoxia in human gastric tumor cells through a protein kinase C-epsilon/ERK/ AP-1 signaling cascade. Cancer Res. 2001, 61 (22), 8322-8330.

(28) (a) Graven, K. K.; McDonald, R. J.; Farber, H. W. Hypoxic regulation of endothelial glyceraldehyde-3-phosphate dehydrogenase. Am. J. Physiol. 1998, 274 (2 Pt 1), C347-C355. (b) Graven, K. K.; Troxler, R. F.; Kornfeld, H.; Panchenko, M. V.; Farber, H. W. Regulation of endothelial cell glyceraldehyde-3-phosphate dehydrogenase expression by hypoxia. J. Biol. Chem. 1994, 269 (39), 2444624453. (c) Li, H.; Ko, H. P.; Whitlock, J. P. Induction of phosphoglycerate kinase 1 gene expression by hypoxia. Roles of Arnt and HIF1alpha. J. Biol. Chem. 1996, 271 (35), 21262-21267. (d) Yamaji, R.; Fujita, K.; Takahashi, S.; Yoneda, H.; Nagao, K.; Masuda, W.; Naito, M.; Tsuruo, T.; Miyatake, K.; Inui, H.; Nakano, Y. Hypoxia up-regulates glyceraldehyde-3-phosphate dehydrogenase in mouse brain capillary endothelial cells: involvement of $\mathrm{Na}+\mathrm{Ca} 2+$ exchanger. Biochim. Biophys. Acta 2003, 1593 (2-3), 269-276.

(29) Marotta, D.; Karar, J.; Jenkins, W. T.; Kumanova, M.; Jenkins, K. W.; Tobias, J. W.; Baldwin, D.; Hatzigeorgiou, A.; Alexiou, P.; Evans, S. M.; Alarcon, R.; Maity, A.; Koch, C.; Koumenis, C. In vivo profiling of 
hypoxic gene expression in gliomas using the hypoxia marker EF5 and laser-capture microdissection. Cancer Res. 2011, 71 (3), 779-789.

(30) Li, D. Q.; Wang, L.; Fei, F.; Hou, Y. F.; Luo, J. M.; Zeng, R.; Wu, J.; Lu, J. S.; Di, G. H.; Ou, Z. L.; Xia, Q. C.; Shen, Z. Z.; Shao, Z. $M$. Identification of breast cancer metastasis-associated proteins in an isogenic tumor metastasis model using two-dimensional gel electrophoresis and liquid chromatography-ion trap-mass spectrometry. Proteomics 2006, 6 (11), 3352-3368.

(31) (a) Semenza, G. L. Targeting HIF-1 for cancer therapy. Nat. Rev. Cancer 2003, 3 (10), 721-732. (b) Wong, C. C.; Zhang, H.; Gilkes, D. M.; Chen, J.; Wei, H.; Chaturvedi, P.; Hubbi, M. E.; Semenza, G. L. Inhibitors of hypoxia-inducible factor 1 block breast cancer metastatic niche formation and lung metastasis. J. Mol. Med. 2012, 90 (7), 803-815. 